\title{
QUASISYMMETRIC FUNCTIONS AND KAZHDAN-LUSZTIG POLYNOMIALS
}

\author{
LOUIS J. BILLERA AND FRANCESCO BRENTI
}

\begin{abstract}
We associate a quasisymmetric function to any Bruhat interval in a general Coxeter group. This association can be seen to be a morphism of Hopf algebras to the subalgebra of all peak functions, leading to an extension of the cd-index of convex polytopes. We show how the Kazhdan-Lusztig polynomial of the Bruhat interval can be expressed in terms of this complete cd-index and otherwise explicit combinatorially defined polynomials. In particular, we obtain the simplest closed formula for the Kazhdan-Lusztig polynomials that holds in complete generality.
\end{abstract}

\section{Contents}

1. Introduction

2. The $R$-quasisymmetric function of a Bruhat interval and the complete cd-index

3. Kazhdan-Lusztig polynomials and the complete cd-index 15

4. Kazhdan-Lusztig polynomials and ballot polynomials 18

5. Other representations of the complete cd-index 22

6. A sign conjecture for the complete cd-index 24

References 26

\section{INTRODUCTION}

The Kazhdan-Lusztig polynomials of a Coxeter group are of fundamental importance in representation theory and in the geometry and topology of Schubert varieties. Defined by means of two separate recursions, they have proved difficult to unravel in any straightforward manner. Here, we reduce their computation to the computation of another invariant of Coxeter groups. This new invariant, a quasisymmetric function that can be

2000 Mathematics Subject Classification. Primary 20F55, 05E99; Secondary 05E15.

Key words and phrases. Coxeter group, Kazhdan-Lusztig polynomial, cd-index, quasisymmetric function.

The first author was supported in part by NSF grants DMS-0100323 and DMS-0555268. The second author was partially supported by EU grant CHRT-CT-2001-00272. This work was begun while both authors enjoyed the hospitality of the Mittag-Leffler Institute, Djursholm, Sweden. 
encoded into a noncommutative polynomial in two variables that we call the complete cd-index, has interesting algebraic and combinatorial properties. We express the Kazhdan-Lusztig polynomial of any Bruhat interval in terms of its complete cd-index and otherwise explicit combinatorially defined polynomials. In particular, we obtain the simplest closed formula for the Kazhdan-Lusztig polynomials that holds in complete generality.

The complete cd-index is defined by means of a quasisysymmetric function $\widetilde{F}(u, v)$ associated to every Bruhat interval $[u, v]$ in a Coxeter group $W$. The association $[u, v] \mapsto \widetilde{F}(u, v)$ can be viewed as a morphism of Hopf algebras, suggesting the possibility of a filtered version of the theory of combinatorial Hopf algebras [1, which predicts the existence of graded maps to the quasisymmetric functions in general combinatorial settings.

The theory of Kazhdan-Lusztig polynomials of Weyl groups is analogous to that of the $g$-polynomials of rational convex polytopes in that they both compute the local intersection cohomology of certain varieties (Schubert and toric, respectively) associated to these objects [28], 31. Also, the recursions that define them in a general Coxeter group (respectively, Eulerian partially ordered set) have the same form. The $g$-polynomial of an Eulerian partially ordered set is known to depend only on the number of chains of certain types. These flag numbers are most succintly represented by the cd-index. On the other hand, for the Kazhdan-Lusztig polynomials it is not even clear that there can be a completely combinatorial description (see, e.g., [7, $\S 5.6]$ ), so there is no straightforward way to generalize this combinatorial description of the $g$-polynomials to the Kazhdan-Lusztig polynomials. Yet, the quasisymmetric functions $\widetilde{F}(u, v)$ do seem to capture much of the spirit of the combinatorial setting of the $g$-polynomials, without themselves being obviously combinatorial.

In the remainder of this section we give the necessary background in Coxeter groups and Kazhdan-Lusztig polynomials and in the use of quasisymmetric functions in poset enumeration. In Section 2 we introduce the $R$-quasisymmetric function of a Bruhat interval, define from this the complete cd-index of the interval and give some of its algebraic properties. Section 3 gives an expression for the Kazhdan-Lusztig polynomials in terms of the complete cd-index and certain lattice path enumerators. Using this expression, we give in Section 4 the representation of the Kazhdan-Lusztig polynomials in terms of an explicit polynomial basis defined by means of the ballot polynomials. The coefficients of this representation are given as linear forms in the complete cd-index. We show by an example that no such representation exists in terms of the ordinary cd-index alone; that is, the Kazhdan-Lusztig polynomials of a Bruhat interval can not be calculated, in general, from the flag $f$-vector of the underlying Eulerian poset. In Section 5 we give a formula for computing the complete cd-index of any Bruhat interval in terms of explicit combinatorial quantities associated to the Bruhat interval. Finally, in Section [6] we conjecture nonnegativity of the complete 
cd-index and show that one consequence of this conjecture holds in the case of finite Coxeter and affine Weyl groups.

1.1. Coxeter groups and Kazhdan-Lusztig polynomials. We follow 25] for general Coxeter groups notation and terminology. In particular, given a Coxeter system $(W, S)$ and $u \in W$ we denote by $l(u)$ the length of $u$ in $W$, with respect to $S$. We denote by $e$ the identity of $W$, and we let $T \stackrel{\text { def }}{=}\left\{u s u^{-1}: u \in W, s \in S\right\}$ be the set of reflections of $W$. We will always assume that $W$ is partially ordered by Bruhat order. Recall (see, e.g., [25, §5.9]) that this means that $x \leq y$ if and only if there exist $r \in \mathbb{N}$ and $t_{1}, \ldots, t_{r} \in T$ such that $t_{r} \cdots t_{1} x=y$ and $l\left(t_{i} \cdots t_{1} x\right)>l\left(t_{i-1} \cdots t_{1} x\right)$ for $i=1, \ldots, r$. Given $u, v \in W$ we let $[u, v] \stackrel{\text { def }}{=}\{x \in W: u \leq x \leq v\}$. We consider $[u, v]$ as a poset with the partial ordering induced by $W$. It is well known (see, e.g., 7], Corollary 2.7.11) that intervals of $W$ (and their duals) are Eulerian posets.

Let $A \subseteq T$ and $W^{\prime}$ be the subgroup of $W$ generated by $A$. Following [25, §8.2], we call $W^{\prime}$ a reflection subgroup of $W$. It is then known (see, e.g., [25], Theorem 8.2) that $\left(W^{\prime}, S^{\prime}\right)$ is again a Coxeter system where $S^{\prime} \stackrel{\text { def }}{=}$ $\left\{t \in T: N(t) \cap W^{\prime}=\{t\}\right\}$, and $N(w) \stackrel{\text { def }}{=}\{t \in T: l(w t)<l(w)\}$. We say that $W^{\prime}$ is a dihedral reflection subgroup if $\left|S^{\prime}\right|=2$ (i.e., if $\left(W^{\prime}, S^{\prime}\right)$ is a dihedral Coxeter system). Following [20] we say that a total ordering $<_{T}$ of $T$ is a reflection ordering if, for any dihedral reflection subgroup $W^{\prime}$ of $W$, we have that either $a<_{T} a b a<_{T} a b a b a<_{T} \cdots<_{T} b a b a b<_{T} b a b<_{T} b$ or $b<_{T} b a b<_{T} b a b a b<_{T} \cdots<_{T} a b a b a<_{T} a b a<_{T} a$ where $\{a, b\} \stackrel{\text { def }}{=} S^{\prime}$. The existence of reflection orderings (and many of their properties) is proved in [20, §2] (see also [7, §5.2]). Throughout this work we will always assume that we have fixed (once and for all) a reflection ordering $<_{T}$ of $T$.

We denote by $\mathcal{H}(W)$ the Hecke algebra associated to $W$. Recall (see, e.g., [25, Chap. 7]) that this is the free $\mathbb{Z}\left[q, q^{-1}\right]$-module having the set $\left\{T_{w}: w \in W\right\}$ as a basis and multiplication such that

$$
T_{w} T_{s}= \begin{cases}T_{w s}, & \text { if } l(w s)>l(w), \\ q T_{w s}+(q-1) T_{w}, & \text { if } l(w s)<l(w),\end{cases}
$$

for all $w \in W$ and $s \in S$. It is well known that this is an associative algebra having $T_{e}$ as unity and that each basis element is invertible in $\mathcal{H}(W)$. More precisely, we have the following result (see [25, Proposition 7.4]).

Proposition 1.1. Let $v \in W$. Then

$$
\left(T_{v^{-1}}\right)^{-1}=q^{-l(v)} \sum_{u \leq v}(-1)^{l(v)-l(u)} R_{u, v}(q) T_{u},
$$

where $R_{u, v}(q) \in \mathbb{Z}[q]$.

The polynomials $R_{u, v}(q)$ defined by the previous proposition are called the $R$-polynomials of $W$. It is easy to see that $R_{u, v}(q)$ is a monic polynomial 
of degree $l(u, v) \stackrel{\text { def }}{=} l(v)-l(u)$, and that $R_{u, u}(q)=1$, for all $u, v \in W, u \leq v$. It is customary to let $R_{u, v}(q) \stackrel{\text { def }}{=} 0$ if $u \not \leq v$. We then have the following fundamental result that follows from (1.1) and Proposition 1.1] (see [25, $\S 7.5])$.

Theorem 1.2. Let $u, v \in W$ and $s \in S$ be such that $l(v s)<l(v)$. Then

$$
R_{u, v}(q)= \begin{cases}R_{u s, v s}(q), & \text { if } l(u s)<l(u), \\ q R_{u s, v s}(q)+(q-1) R_{u, v s}(q), & \text { if } l(u s)>l(u) .\end{cases}
$$

Note that the preceding theorem can be used to inductively compute the $R$-polynomials. Theorem 1.2 has also the following simple but important consequence (see, for example, [7, Proposition 5.3.1]).

Proposition 1.3. Let $u, v \in W$. Then there exists a (necessarily unique) polynomial $\widetilde{R}_{u, v}(q) \in \mathbb{N}[q]$ such that

$$
R_{u, v}(q)=q^{\frac{1}{2}(l(v)-l(u))} \widetilde{R}_{u, v}\left(q^{\frac{1}{2}}-q^{-\frac{1}{2}}\right) .
$$

Combinatorial interpretations of the coefficients of $\widetilde{R}_{u, v}(q)$ have been given by V. Deodhar [18] and by M. Dyer [20] (see [7, Theorem 5.3.7] and [7, Theorem 5.3.4]).

The $R$-polynomials can be used to define the Kazhdan-Lusztig polynomials. The following result is not hard to prove (and, in fact, holds in much greater generality, see [32, Corollary 6.7] and [32, Example 6.9]) and a proof can be found, e.g., in [25, §7.9-11] or [27, §2.2].

Theorem 1.4. There is a unique family of polynomials $\left\{P_{u, v}(q)\right\}_{u, v \in W} \subseteq$ $\mathbb{Z}[q]$, such that, for all $u, v \in W$,

1. $P_{u, v}(q)=0$ if $u \not \leq v$;

2. $P_{u, u}(q)=1$;

3. $\operatorname{deg}\left(P_{u, v}(q)\right)<\frac{1}{2}(l(v)-l(u))$, if $u<v$;

4.

$$
q^{l(v)-l(u)} P_{u, v}\left(\frac{1}{q}\right)=\sum_{u \leq z \leq v} R_{u, z}(q) P_{z, v}(q),
$$

if $u \leq v$.

The polynomials $P_{u, v}(q)$ defined by the preceding theorem are called the Kazhdan-Lusztig polynomials of $W$.

1.2. Paths in Bruhat graphs. Recall that a composition of a positive integer $n$ is a finite sequence of positive integers $\alpha=\alpha_{1} \cdots \alpha_{k}$ such that $\sum_{i} \alpha_{i}=n$. In this case we write $\alpha \models n$ and we define $l(\alpha)=k,|\alpha|=n$, and $\alpha^{*}=\alpha_{k} \cdots \alpha_{1}$. (There should be no confusion with our using the same notation $l(\cdot)$ for the lengths of compositions and elements of $W$.) Given two compositions $\alpha_{1} \cdots \alpha_{s}, \beta_{1} \cdots \beta_{t}$ of $n$ we say that $\alpha_{1} \cdots \alpha_{s}$ refines $\beta_{1} \cdots \beta_{t}$ if there exist $1 \leq i_{1}<i_{2}<\cdots<i_{t-1}<s$ such that $\sum_{j=i_{k-1}+1}^{i_{k}} \alpha_{j}=\beta_{k}$ for 
$k=1, \ldots, t$ (where $\left.i_{0} \stackrel{\text { def }}{=} 0, i_{t} \stackrel{\text { def }}{=} s\right)$. We then write $\alpha_{1} \cdots \alpha_{s} \preceq \beta_{1} \cdots \beta_{t}$. We denote by $C$ the set of all finite sequences of positive integers (i.e., the set of all compositions).

Recall (see [25], §8.6, or [19]) that the Bruhat graph of a Coxeter system $(W, S)$ is the directed graph $B(W, S)$ obtained by taking $W$ as vertex set and putting a directed edge from $x$ to $y$ if and only if $y x^{-1} \in T$ and $l(x)<$ $l(y)$. We call the directed paths of $B(W, S)$ Bruhat paths. These should be distinguished from chains in the Bruhat order. The vertex set of a Bruhat path is always a chain, but not all chains form Bruhat paths.

Given a Bruhat path $\Delta=\left(a_{0}, a_{1}, \ldots, a_{r}\right)$ from $a_{0}$ to $a_{r}$, we define its length to be $l(\Delta) \stackrel{\text { def }}{=} r$, its descent set, with respect to $<_{T}$, to be

$$
D(\Delta) \stackrel{\text { def }}{=}\left\{i \in[r-1]: a_{i}\left(a_{i-1}\right)^{-1}>_{T} a_{i+1}\left(a_{i}\right)^{-1}\right\},
$$

and its descent composition to be the unique composition $\mathcal{D}(\Delta)$ of $r$ corresponding to $D(\Delta)$ under the usual bijection between compositions and sets $\beta_{1} \beta_{2} \cdots \beta_{k} \mapsto\left\{\beta_{1}, \beta_{1}+\beta_{2}, \ldots, \beta_{1}+\cdots+\beta_{k-1}\right\}$. We will denote the inverse of this bijection by $c o(\cdot)$. Given $u, v \in W$, and $k \in \mathbb{N}$, we denote by $B_{k}(u, v)$ the set of all directed paths in $B(W, S)$ from $u$ to $v$ of length $k$, and we let $B(u, v) \stackrel{\text { def }}{=} \bigcup_{k \geq 0} B_{k}(u, v)$. For $u, v \in W$, and $\alpha \in C$, we let, following [11,

$$
c_{\alpha}(u, v) \stackrel{\text { def }}{=}\left|\left\{\Delta \in B_{|\alpha|}(u, v): \mathcal{D}(\Delta) \succeq \alpha\right\}\right|,
$$

and

$$
b_{\alpha}(u, v) \stackrel{\text { def }}{=}\left|\left\{\Delta \in B_{|\alpha|}(u, v): \mathcal{D}(\Delta)=\alpha\right\}\right| .
$$

Note that these definitions imply that

$$
c_{\alpha}(u, v)=\sum_{\{\beta \models n: \beta \succeq \alpha\}} b_{\beta}(u, v)
$$

for all $u, v \in W$ and $\alpha \models n(n \in \mathbb{P})$.

The following result follows from [11, Proposition 4.4]. Given a polynomial $P(q)$, and $i \in \mathbb{Z}$, we denote by $\left[q^{i}\right](P)$ the coefficient of $q^{i}$ in $P(q)$.

Proposition 1.5. Let $u, v \in W, u \leq v$, and $\alpha \in C$. Then

$$
c_{\alpha}(u, v)=\sum_{\left(u_{0}, \ldots, u_{r}\right) \in C_{r}(u, v)} \prod_{j=1}^{r}\left[q^{\alpha_{j}}\right]\left(\widetilde{R}_{u_{j-1}, u_{j}}\right)
$$

where $C_{r}(u, v)$ denotes the set of all chains of length $r$ (totally ordered subsets of cardinality $r+1)$ from $u$ to $v$, and $r \stackrel{\text { def }}{=} l(\alpha)$.

Proposition 1.5 shows, in particular, that $c_{\alpha}(u, v)$ (and hence $b_{\alpha}(u, v)$ ) are independent of the total reflection ordering $<_{T}$ used to define them.

For $j \in \mathbb{Q}$ we define an operator $D_{j}: \mathbb{R}[q] \rightarrow \mathbb{R}[q]$ by letting

$$
D_{j}\left(\sum_{i \geq 0} a_{i} q^{i}\right) \stackrel{\text { def }}{=} \sum_{i=0}^{\lfloor j\rfloor} a_{i} q^{i} .
$$


For $\alpha \in C$ we define, following [12, a polynomial $\Psi_{\alpha}(q) \in \mathbb{Z}[q]$ inductively as follows,

$$
\Psi_{\alpha}(q) \stackrel{\text { def }}{=}(q-1)^{\alpha_{1}} D_{\frac{\left|\alpha^{-}\right|-1}{2}}\left(\Psi_{\alpha^{-}}(q)\right)
$$

if $l(\alpha) \geq 2$, and

$$
\Psi_{\alpha}(q) \stackrel{\text { def }}{=}(q-1)^{|\alpha|},
$$

if $l(\alpha)=1$. Here $\alpha^{-}=\alpha_{2} \cdots \alpha_{k}$ when $\alpha=\alpha_{1} \cdots \alpha_{k}$. For $n \in \mathbb{P}$ and $\beta \models n$ we then let

$$
\Upsilon_{\beta}(q) \stackrel{\text { def }}{=} \sum_{\{\alpha \models n: \alpha \preceq \beta\}}(-1)^{l(\alpha)} \Psi_{\alpha}(q) .
$$

We then have the following result, whose proof can be found in $[7$, Theorem 5.5.7].

Theorem 1.6. Let $u, v \in W, u<v$. Then

$$
P_{u, v}(q)-q^{l(u, v)} P_{u, v}\left(\frac{1}{q}\right)=\sum_{\beta \in C} q^{\frac{l(u, v)-|\beta|}{2}} \Upsilon_{\beta}(q) b_{\beta}(u, v) .
$$

Let $\left(W_{1}, S_{1}\right)$ and $\left(W_{2}, S_{2}\right)$ be two Coxeter systems. It is clear that the direct product $W=W_{1} \times W_{2}$ is again a Coxeter group with respect to the generating set $S=S_{1} \sqcup S_{2}$ (disjoint union), having Dynkin diagram the disjoint union of the diagrams of $\left(W_{1}, S_{1}\right)$ and $\left(W_{2}, S_{2}\right)$. It then follows immediately from the subword property of Bruhat order (see, e.g., [7, Theorem 2.2.2]) that $W$, as a poset under Bruhat order, is isomorphic to the direct product of $W_{1}$ and $W_{2}$ as posets under Bruhat order. Thus $\left(u_{1}, u_{2}\right) \leq\left(v_{1}, v_{2}\right)$ in $W$ if and only if $u_{1} \leq v_{1}$ in $W_{1}$ and $u_{2} \leq v_{2}$ in $W_{2}$. Thus Theorems 1.2 and 1.4 imply the following multiplicative formulas. We include a proof for lack of an adequate reference.

Proposition 1.7. Let $u_{1}, v_{1} \in W_{1}$ and $u_{2}, v_{2} \in W_{2}$. Then

$$
\begin{aligned}
& R_{\left(u_{1}, u_{2}\right),\left(v_{1}, v_{2}\right)}=R_{u_{1}, v_{1}} \cdot R_{u_{2}, v_{2}}, \text { and } \\
& P_{\left(u_{1}, u_{2}\right),\left(v_{1}, v_{2}\right)}=P_{u_{1}, v_{1}} \cdot P_{u_{2}, v_{2}} .
\end{aligned}
$$

Proof. First note that by the comments preceding this proposition, (1.5) and (1.6) hold if $\left(u_{1}, u_{2}\right) \not \leq\left(v_{1}, v_{2}\right)$, so assume that $\left(u_{1}, u_{2}\right) \leq\left(v_{1}, v_{2}\right)$. We first prove (1.5) by induction on $l\left(v_{2}\right)$.If $v_{2}=e$ then $u_{2}=e$ and (1.5) follows from Theorem 1.2 and the fact that $D((u, e))=\{(s, e): s \in D(u)\}$ for all $u \in W_{1}$. (Note that $l_{W}\left(\left(u_{1}, u_{2}\right),\left(v_{1}, v_{2}\right)\right)=l_{W_{1}}\left(u_{1}, v_{1}\right)+l_{W_{2}}\left(u_{2}, v_{2}\right)$.) If $v_{2}>e$ and $s \in D\left(v_{2}\right)$, then $(e, s) \in D\left(\left(v_{1}, v_{2}\right)\right)$ and therefore, by Theorem 1.2 and our induction hypothesis,

$$
\begin{aligned}
R_{\left(u_{1}, u_{2}\right),\left(v_{1}, v_{2}\right)} & =q R_{\left(u_{1}, u_{2} s\right),\left(v_{1}, v_{2} s\right)}+(q-1) R_{\left(u_{1}, u_{2}\right),\left(v_{1}, v_{2} s\right)} \\
& =R_{u_{1}, v_{1}}\left(q R_{u_{2} s, v_{2} s}+(q-1) R_{u_{2}, v_{2} s}\right) \\
& =R_{u_{1}, v_{1}} \cdot R_{u_{2}, v_{2}}
\end{aligned}
$$


if $s \notin D\left(u_{2}\right)$ (so $\left.(e, s) \notin D\left(\left(u_{1}, u_{2}\right)\right)\right)$, while

$$
\begin{aligned}
R_{\left(u_{1}, u_{2}\right),\left(v_{1}, v_{2}\right)}=R_{\left(u_{1}, u_{2} s\right),\left(v_{1}, v_{2} s\right)} & =R_{u_{1}, v_{1}} R_{u_{2} s, v_{2} s} \\
& =R_{u_{1}, v_{1}} R_{u_{2}, v_{2}}
\end{aligned}
$$

if $s \in D\left(u_{2}\right)$, as desired.

We conclude by proving (1.6) by induction on $l_{W}\left(\left(u_{1}, u_{2}\right),\left(v_{1}, v_{2}\right)\right)$. The result is clear if $l_{W}\left(\left(u_{1}, u_{2}\right),\left(v_{1}, v_{2}\right)\right)=0$. If $l_{W}\left(\left(u_{1}, u_{2}\right),\left(v_{1}, v_{2}\right)\right)>0$ then by Theorem 1.4, our induction hypothesis, (1.5) and the comments preceding this proposition, we have

$$
\begin{aligned}
& q^{l\left(\left(u_{1}, u_{2}\right),\left(v_{1}, v_{2}\right)\right)} P_{\left(u_{1}, u_{2}\right),\left(v_{1}, v_{2}\right)}\left(\frac{1}{q}\right)-P_{\left(u_{1}, u_{2}\right),\left(v_{1}, v_{2}\right)}(q) \\
& =\sum_{\left(u_{1}, u_{2}\right)<\left(x_{1}, x_{2}\right) \leq\left(v_{1}, v_{2}\right)} R_{\left(u_{1}, u_{2}\right),\left(x_{1}, x_{2}\right)}(q) P_{\left(x_{1}, x_{2}\right),\left(v_{1}, v_{2}\right)}(q) \\
& =\sum_{u_{1} \leq x_{1} \leq v_{1}} R_{u_{1}, x_{1}}(q) P_{x_{1}, v_{1}}(q) \sum_{\substack{u_{2} \leq x_{2} \leq v_{2}\\
}} R_{u_{2}, x_{2}}(q) P_{x_{2}, v_{2}}(q) \\
& =P_{u_{1}, v_{1}}(q) P_{u_{2}, v_{2}}(q) \\
& =q^{l\left(u_{1}, v_{1}\right)} P_{u_{1}, v_{1}}\left(\frac{1}{q}\right) q^{l\left(u_{2}, v_{2}\right)} P_{u_{2}, v_{2}}\left(\frac{1}{q}\right)-P_{u_{1}, v_{1}}(q) P_{u_{2}, v_{2}}(q),
\end{aligned}
$$

and (1.6) follows.

Throughout this work (unless otherwise explicitly stated) $(W, S)$ denotes a fixed (but arbitrary) Coxeter system.

1.3. Quasisymmetric functions and poset enumeration. A quasisymmetric function is a formal power series in countably many variables that has bounded degree and whose coefficients are invariant under shifts of the variables that respect their order. We assume here that the reader is familiar with the basics of the theory of quasisymmetric functions, for example, as described in $[34, \S 7.19]$. We denote by $Q S y m \subset \mathbb{Q}\left[\left[x_{1}, x_{2}, \ldots\right]\right]$ the algebra of all quasisymmetric functions (with rational coefficients). QSym is a graded algebra with the usual grading of power series; we denote by $\mathcal{Q}_{i}$ the $\mathrm{i}^{\text {th }}$ homogeneous part of $Q S y m$ and so

$$
Q S y m=\mathcal{Q}_{0} \oplus \mathcal{Q}_{1} \oplus \cdots .
$$

In particular, we will make use of the monomial basis $\left\{M_{\alpha}\right\}_{\alpha \in C}$ and the fundamental basis $\left\{L_{\alpha}\right\}_{\alpha \in C}$ for QSym, where for a composition $\alpha=$ $\alpha_{1} \alpha_{2} \cdots \alpha_{k}, k>0, \alpha_{i}>0$,

$$
M_{\alpha}=\sum_{i_{1}<i_{2}<\cdots<i_{k}} x_{i_{1}}^{\alpha_{1}} x_{i_{2}}^{\alpha_{2}} \cdots x_{i_{k}}^{\alpha_{k}}
$$

and

$$
L_{\beta}=\sum_{\{\alpha \models|\beta|: \alpha \preceq \beta\}} M_{\alpha} .
$$


We include as well the empty composition $\alpha=\mathbf{0}$ (the case $k=0$ ); here we set $M_{\mathbf{0}}=L_{\mathbf{0}}=1$. Note that the degree of $M_{\alpha}$ and $L_{\alpha}$ is $|\alpha|$. Occasionally, it will be useful to index these bases by subsets of $[n-1]$ instead of compositions of $n$, using the standard bijection between compositions and subsets already mentioned. In this case, we write $L_{T}^{(n)}$ to indicate its degree.

An interesting subalgebra of QSym is the subspace $\Pi$ of peak functions, which can be defined as follows. Let $\mathbf{c}$ and $\mathbf{d}$ be noncommuting indeterminates of degree 1 and 2, respectively. We let $w$ be an arbitrary word in the letters $\mathbf{c}$ and $\mathbf{d}$. If

$$
w=\mathbf{c}^{n_{1}} \mathbf{d} \mathbf{c}^{n_{2}} \mathbf{d} \cdots \mathbf{c}^{n_{k}} \mathbf{d} \mathbf{c}^{n_{0}},
$$

$\left(n_{0}, \ldots, n_{k} \geq 0\right)$ then let $m_{j}=\operatorname{deg}\left(\mathbf{c}^{n_{1}} \mathbf{d} \mathbf{c}^{n_{2}} \mathbf{d} \cdots \mathbf{c}^{n_{j}} \mathbf{d}\right), j=1, \ldots, k$. Define $\mathcal{I}^{w}$ to be the family consisting of the $k$ 2-element subsets $\left\{m_{j}-1, m_{j}\right\}$, $j=1, \ldots, k$, and

$$
b\left[\mathcal{I}^{w}\right] \stackrel{\text { def }}{=}\left\{T \subseteq[n]: S \cap T \neq \emptyset, \text { for all } S \in \mathcal{I}^{w}\right\},
$$

where $\mathrm{n}=\operatorname{deg}(w)$. Finally, define

$$
\Theta_{w} \stackrel{\text { def }}{=} \sum_{T \in B\left[\mathcal{I}^{w}\right]} L_{T}^{(n+1)},
$$

where $B\left[\mathcal{I}^{w}\right] \stackrel{\text { def }}{=}\left\{T \subseteq[n]: T, \bar{T} \in b\left[\mathcal{I}^{w}\right]\right\}$. Note that $\operatorname{deg}\left(\Theta_{w}\right)=\operatorname{deg}(w)+1$. Here, if $w=\mathbf{1}$, the empty cd-word, then $\Theta_{1}=L_{\emptyset}^{(1)}=L_{1}$. We can define $\Pi$ to be the linear subspace of QSym spanned by 1 and all the $\Theta_{w}$, as $w$ ranges over all cd words. Again, $\Pi$ is a graded algebra with the inherited grading; we denote by $\Pi_{i} \stackrel{\text { def }}{=} \Pi \cap \mathcal{Q}_{i}$ its $i^{\text {th }}$ homogeneous part. See [6] and [35] for details. There the basis element $\Theta_{w}$ in (1.8) is replaced by $\Theta_{w}^{(s t)}=2^{|w|_{\mathbf{d}}+1} \Theta_{w}$, where we denote the degree of $w$ by $|w|$ and extend this notation to let $|w|_{\mathbf{d}}$ denote the number of d's in $w$.

We summarize here the basics of the use of quasisymmetric functions in the theory of flag $f$-vectors of graded posets and, in particular, Eulerian posets. For a finite graded poset $Q$, with rank function $\rho(\cdot)$, we define the formal power series

$$
F(Q) \stackrel{\text { def }}{=} \sum_{\hat{0}=u_{0} \leq \cdots \leq u_{k-1}<u_{k}=\hat{1}} x_{1}^{\rho\left(u_{0}, u_{1}\right)} x_{2}^{\rho\left(u_{1}, u_{2}\right)} \cdots x_{k}^{\rho\left(u_{k-1}, u_{k}\right)},
$$

where the sum is over all multichains in $Q$ whose last two elements are different and $\rho(x, y)=\rho(y)-\rho(x)$. For general properties of $F(Q)$, see [21] and [6]. In particular, we have the following

Proposition 1.8. For a graded poset $Q$,

1. $F(Q) \in Q$ Sym and is homogeneous of degree $\rho(Q)$,

2. $F\left(Q_{1} \times Q_{2}\right)=F\left(Q_{1}\right) F\left(Q_{2}\right)$,

3. $F(Q)=\sum_{\alpha} f_{\alpha} M_{\alpha}=\sum_{\alpha} h_{\alpha} L_{\alpha}$, where $f_{\alpha}$ and $h_{\alpha}$ are the flag $f$ and flag h-vectors, respectively, of $Q$, and 
4. If $Q$ is Eulerian, then $F(Q) \in \Pi$; in fact $F(Q)=\sum_{w}[w]_{Q} \Theta_{w}$, where $[w]_{Q}$ denotes the coefficient of $w$ in the $\mathbf{c d}$-index of $Q$.

The last statement of Proposition 1.8, which follows from Proposition 2.2 and Theorem 2.1 of [6], can be used as the definition of the cd-index for an Eulerian poset $Q$. Formally, the cd-index is the homogeneous noncommutative polynomial, $\psi_{Q}=\psi_{Q}(\mathbf{c}, \mathbf{d})=\sum_{w}[w]_{Q} w$ in $\mathbf{c}$ and $\mathbf{d}$, where the sum is over all cd-words of degree $\rho(Q)-1$; see [4].

\section{The $R$-Quasisymmetric Function of a Bruhat interval And THE COMPLETE cd-INDEX}

Since a Bruhat interval $[u, v]$ is an Eulerian poset, it has a homogeneous cd-index $\psi_{u, v} \stackrel{\text { def }}{=} \psi_{[u, v]}$ and quasisymmetric function $F(u, v) \stackrel{\text { def }}{=} F([u, v])$ defined as above. The polynomial $\psi_{u, v}$ has been studied explicitly by Reading [30. In this section, we extend the definition of the cd-index for Bruhat intervals to get a nonhomogeneous polynomial, whose coefficients we later use to give a simple expression for Kazhdan-Lusztig polynomials.

2.1. The $R$-quasisymmetric function of a Bruhat interval. We define a quasisymmetric function, analogous to the power series (1.9), making use of the polynomials $\widetilde{R}_{u, v}$ defined in Proposition 1.3 .

Given $u, v \in W, u \leq v$, we define the R-quasisymmetric function $\widetilde{F}(u, v)$ by

$$
\widetilde{F}(u, v) \stackrel{\text { def }}{=} \sum_{u=u_{0} \leq \cdots \leq u_{k-1}<u_{k}=v} \widetilde{R}_{u_{0} u_{1}}\left(x_{1}\right) \widetilde{R}_{u_{1} u_{2}}\left(x_{2}\right) \cdots \widetilde{R}_{u_{k-1} u_{k}}\left(x_{k}\right),
$$

where, again, the sum is over all multichains in $[u, v]$ whose last two elements are different. Note that, by Proposition 1.3 and the comments following Proposition 1.1, the leading term of each summand on the right hand side of (2.1) is the corresponding monomial on the right hand side of (1.9).

An alternative description of $\widetilde{F}(u, v)$ in terms of chains is as follows. We omit the straightforward verification.

Proposition 2.1. Let $u, v \in W, u<v$. Then

$$
\widetilde{F}(u, v)=\sum_{k \geq 1} \sum_{u=u_{0}<u_{1}<\cdots<u_{k}=v} \sum_{1 \leq i_{1}<i_{2}<\cdots<i_{k}} \prod_{j=1}^{k} \widetilde{R}_{u_{j-1}, u_{j}}\left(x_{i_{j}}\right) .
$$

We will see that $\widetilde{F}(u, v)$ shares many of the properties of $F(Q)$ and will serve to define an extension of the cd-index for Bruhat intervals. In particular, we have the following

Theorem 2.2. For any $u, v \in W, u \leq v$,

1. $\widetilde{F}(u, v)=\sum_{\alpha} c_{\alpha}(u, v) M_{\alpha}=\sum_{\alpha} b_{\alpha}(u, v) L_{\alpha}$, and 
2. $\widetilde{F}(u, v) \in \Pi$, in fact

$$
\widetilde{F}(u, v) \in \Pi_{l(u, v)} \oplus \Pi_{l(u, v)-2} \oplus \Pi_{l(u, v)-4} \oplus \cdots .
$$

Proof. To prove part 1, we have, using Proposition 1.5,

$$
\begin{aligned}
\widetilde{F}(u, v) & =\sum_{u=u_{0} \leq \cdots \leq u_{k-1}<u_{k}=v} \widetilde{R}_{u_{0} u_{1}}\left(x_{1}\right) \widetilde{R}_{u_{1} u_{2}}\left(x_{2}\right) \cdots \widetilde{R}_{u_{k-1} u_{k}}\left(x_{k}\right) \\
& =\sum_{u=u_{0} \leq \cdots \leq u_{k-1}<u_{k}=v} \prod_{j=1}^{k} \sum_{\alpha_{j} \geq 0}\left(x_{j}^{\alpha_{j}}\left[q^{\alpha_{j}}\right]\left(\widetilde{R}_{u_{j-1}, u_{j}}\right)\right) \\
& =\sum_{u=u_{0} \leq \cdots \leq u_{k-1}<u_{k}=v} \sum_{\alpha \in \mathbb{N}^{k}} \prod_{j=1}^{k}\left(x_{j}^{\alpha_{j}}\left[q^{\alpha_{j}}\right]\left(\widetilde{R}_{u_{j-1}, u_{j}}\right)\right) \\
& =\sum_{k \geq 1} \sum_{\left\{\alpha \in \mathbb{N}^{k}: \alpha_{k}>0\right\}} x_{1}^{\alpha_{1}} \cdots x_{k}^{\alpha_{k}} \sum_{u=u_{0} \leq \cdots \leq u_{k-1}<u_{k}=v} \prod_{j=1}^{k}\left[q^{\alpha_{j}}\right]\left(\widetilde{R}_{u_{j-1}, u_{j}}\right) \\
& =\sum_{k \geq 1} \sum_{\left\{\alpha \in \mathbb{N}^{k}: \alpha_{k}>0\right\}} x_{1}^{\alpha_{1}} \cdots x_{k}^{\alpha_{k}} \sum_{u=u_{0}<\cdots<u_{l(\alpha+)}=v} \prod_{j=1}^{\left.\alpha_{j}^{+}\right]}\left(\widetilde{R}_{u_{j-1}, u_{j}}\right) \\
& =\sum_{k \geq 1} \sum_{\left\{\alpha \in \mathbb{N}^{k}: \alpha_{k}>0\right\}} x_{1}^{\alpha_{1}} \cdots x_{k}^{\alpha_{k}} c_{\alpha^{+}}(u, v) \\
& =\sum_{\beta \in C} c_{\beta}(u, v) M_{\beta},
\end{aligned}
$$

where we have used the facts that $\left[q^{0}\right]\left(\widetilde{R}_{x, y}\right)=\delta_{x, y}$ for all $x, y \in W, x \leq y$, and $\widetilde{R}_{x, x}=1$. Here $\alpha^{+}$is the composition obtained by taking only positive entries of $\alpha$ in order. The second equality in part 1 follows from (1.4) and (1.7).

Part 2 now follows easily from [12, Theorem 8.4] and [6, Proposition 1.3]. The last assertion follows since the $c_{\alpha}(u, v)$ count certain directed paths from $u$ to $v$ of length $|\alpha|$ in the Bruhat graph $B(W, S)$, and all of these must have length $\equiv l(u, v)(\bmod 2)$.

As a consequence of Theorem 2.2, we can express any $\widetilde{F}(u, v)$ in terms of the basis $\Theta_{w}$ for $\Pi$.

Corollary 2.3. For any $u, v \in W, u \leq v$, we can write

$$
\widetilde{F}(u, v)=\sum_{w}[w]_{u, v} \Theta_{w} .
$$

Note that, by Theorem $\left[2.2\right.$, the coefficients $[w]_{u, v}$ can be nonzero only when $\operatorname{deg}(w)=l(u, v)-1, l(u, v)-3, \ldots$ We find it convenient to define, 
for any $u, v \in W$,

$$
\widetilde{\psi}_{u, v}=\widetilde{\psi}_{u, v}(\mathbf{c}, \mathbf{d})=\sum_{w}[w]_{u, v} w
$$

a nonhomogeneous, noncommutative polynomial in the variables $\mathbf{c}$ and $\mathbf{d}$ that will be called the complete $\mathbf{c d}$-index of the Bruhat interval $[u, v]$.

Example 2.4. Let $W=S_{4}, u=1234$ and $v=4231$. Choose the reflection ordering $(1,2)<(1,3)<(1,4)<(2,3)<(2,4)<(3,4)$. Then there are 73 Bruhat paths from $u$ to $v$ and from (1.3) one can compute that

$$
\begin{aligned}
& b_{5}(u, v)=b_{1,1,1,1,1}(u, v)=b_{1}(u, v)=1, \\
& b_{3}(u, v)=b_{2,1}(u, v)=b_{1,2}(u, v)=b_{1,1,1}(u, v)=b_{1,4}(u, v)=b_{2,1,1,1}(u, v)=2, \\
& b_{4,1}(u, v)=b_{3,1,1}(u, v)=b_{1,1,3}(u, v)=b_{1,1,1,2}(u, v)=3 \\
& b_{2,3}(u, v)=b_{1,2,1,1}(u, v)=4 \\
& b_{3,2}(u, v)=b_{1,1,2,1}(u, v)=5 \\
& b_{1,3,1}(u, v)=b_{2,1,2}(u, v)=6, \text { and } \\
& b_{2,2,1}(u, v)=b_{1,2,2}(u, v)=8
\end{aligned}
$$

so by Theorem 2.2

$$
\begin{aligned}
\widetilde{F}(1234,4231)= & L_{5}+3 L_{4,1}+5 L_{3,2}+4 L_{2,3}+2 L_{1,4} \\
& +3 L_{3,1,1}+6 L_{1,3,1}+3 L_{1,1,3}+8 L_{2,2,1}+6 L_{2,1,2}+8 L_{1,2,2} \\
& +2 L_{2,1,1,1}+4 L_{1,2,1,1}+5 L_{1,1,2,1}+3 L_{1,1,1,2}+L_{1,1,1,1,1} \\
& +2 L_{3}+2 L_{2,1}+2 L_{1,2}+2 L_{1,1,1}+L_{1} \\
= & \Theta_{\mathbf{c}^{4}}+\Theta_{\mathbf{d c}^{2}}+2 \Theta_{\mathbf{c d c}}+2 \Theta_{\mathbf{c}^{2} \mathbf{d}}+2 \Theta_{\mathbf{d}^{2}}+2 \Theta_{\mathbf{c}^{2}}+\Theta_{\mathbf{1}}
\end{aligned}
$$

and so $\left[\mathbf{d c}^{2}\right]_{1234,4231}=1,[\mathbf{c d c}]_{1234,4231}=2$, etc. Thus we have

$$
\widetilde{\psi}_{1234,4231}=\mathbf{c}^{4}+\mathbf{d} \mathbf{c}^{2}+2 \mathbf{c d} \mathbf{c}+2 \mathbf{c}^{2} \mathbf{d}+2 \mathbf{d}^{2}+2 \mathbf{c}^{2}+\mathbf{1}
$$

There is at least one case where (1.9) and (2.1) define the same element of QSym. For $u, v \in W$ and $i \in \mathbb{N}$ let $\widetilde{F}_{i}(u, v)$ be the homogeneous component of $\widetilde{F}(u, v)$ of degree $i$ (so $\widetilde{F}_{i}(u, v)=0$ unless $\left.i \equiv l(u, v)(\bmod 2)\right)$.

Proposition 2.5. Let $u, v \in W, u \leq v$. Then $\widetilde{F}_{l(u, v)}(u, v)=F(u, v)$ and so $[w]\left(\widetilde{\psi}_{u, v}\right)=[w]\left(\psi_{u, v}\right)$ when $\operatorname{deg}(w)=l(u, v)-1$. In particular, $\widetilde{F}(u, v)=$ $F(u, v)$ and $\widetilde{\psi}_{u, v}=\psi_{u, v}$ whenever the Bruhat interval $[u, v]$ is a lattice.

Proof. The first assertion follows immediately from (1.9), (2.1) and the comments following Proposition 1.1, The second one follows from (1.9), (2.1) and [10, Corollary 6.5].

2.2. Algebraic properties of $\widetilde{F}$. We investigate some of the algebraic properties of the map $[u, v] \mapsto \widetilde{F}(u, v)$. In [21, Proposition 4.4], the map on posets, $P \mapsto F(P)$, is shown to be a morphism of Hopf algebras. The same 
holds for the map defined by $\widetilde{F}$. To see this, note that the coproduct on posets used in [21] restricts to one on Bruhat intervals:

$$
\Delta([u, v])=\sum_{w \in[u, v]}[u, w] \otimes[w, v] .
$$

On QSym, we take the usual coproduct defined by

$$
\Delta\left(M_{\gamma}\right)=\sum_{\alpha \cdot \beta=\gamma} M_{\alpha} \otimes M_{\beta}
$$

where $\alpha \cdot \beta$ denotes concatenation of compositions.

We begin with an analog of Proposition 1.8.2, which shows the map to be multiplicative with respect to direct product.

Proposition 2.6. Let $W_{1}, W_{2}$ be two Coxeter groups, $W_{1} \times W_{2}$ be their direct product, and $u_{1}, v_{1} \in W_{1}, u_{2}, v_{2} \in W_{2}$. Then

$$
\widetilde{F}\left(\left(u_{1}, u_{2}\right),\left(v_{1}, v_{2}\right)\right)=\widetilde{F}\left(u_{1}, v_{1}\right) \widetilde{F}\left(u_{2}, v_{2}\right) .
$$

Proof. It follows from Proposition [1.7 and Proposition [1.3 that for $u_{1} \leq$ $z_{1} \leq z_{2} \leq v_{1}$ and $u_{2} \leq w_{1} \leq w_{2} \leq v_{2}$

$$
\widetilde{R}_{\left(z_{1}, w_{1}\right),\left(z_{2}, w_{2}\right)}=\widetilde{R}_{z_{1}, z_{2}} \widetilde{R}_{w_{1}, w_{2}}
$$

The proof then follows by the same limiting argument used to prove multiplicativity of the map $F$ [21, Proposition 4.4], with the change that we now use the maps $\widetilde{\kappa}_{i}(u, v)=\widetilde{R}_{u, v}\left(x_{i}\right)$, which are multiplicative by (2.4). We omit the details.

That $\widetilde{F}$ is a coalgebra map is proved next.

Proposition 2.7. The map $\widetilde{F}$ is a map of coalgebras, that is, for each $u, v \in W, u \leq v$

$$
((\widetilde{F} \otimes \widetilde{F}) \circ \Delta)([u, v])=\Delta(\widetilde{F}(u, v)) .
$$

Proof. By Theorem 2.2.1 and (2.3), we can write

$$
\begin{aligned}
\Delta(\widetilde{F}(u, v)) & =\sum_{\gamma} c_{\gamma}(u, v) \sum_{\alpha \cdot \beta=\gamma} M_{\alpha} \otimes M_{\beta} \\
& =\sum_{\alpha} \sum_{\beta} c_{\alpha \cdot \beta}(u, v) M_{\alpha} \otimes M_{\beta} .
\end{aligned}
$$

Now, as an extension of [2, Proposition 5.5.4] (and with virtually the same proof), we have $c_{\alpha \cdot \beta}(u, v)=\sum_{w \in[u, v]} c_{\alpha}(u, w) c_{\beta}(w, v)$. Thus

$$
\begin{aligned}
\Delta(\widetilde{F}(u, v)) & =\sum_{w \in[u, v]} \sum_{\alpha} \sum_{\beta} c_{\alpha}(u, w) c_{\beta}(w, v) M_{\alpha} \otimes M_{\beta} \\
& =\sum_{w \in[u, v]} \widetilde{F}(u, w) \otimes \widetilde{F}(w, v),
\end{aligned}
$$


completing the proof.

Remark 2.8. Formally, let $\mathcal{C}$ be the graded vector space, over a field $\mathbf{k}$, spanned by $1 \in \mathbf{k}$ and all isomorphism classes of Bruhat intervals $[u, v]$, $u<v$, in all Coxeter groups, where the elements of $\mathbf{k}$ have degree 0 and $\operatorname{deg}([u, v])=l(v)-l(u)$, and where $[u, v] \cong[w, z]$ if there exists a directed graph isomorphism $f:[u, v] \rightarrow[w, z]$ such that $D(\Delta)=D(f(\Delta))$ for all Bruhat paths $\Delta$ in $[u, v]$. $\mathcal{C}$ has multiplication defined via Cartesian product $\left[u_{1}, v_{1}\right] \times\left[u_{2}, v_{2}\right]=\left[\left(u_{1}, u_{2}\right),\left(v_{1}, v_{2}\right)\right]$, and comultiplication defined by $\Delta([u, v])=\sum_{w \in[u, v]}[u, w] \otimes[w, v]$, where $[u, u]$ is defined to be $1 \in \mathbf{k}$. We define a counit $\epsilon$ on $\mathcal{C}$ by $\epsilon([u, v])=\delta_{0, l(v)-l(u)}$ for $u \leq v$. By [21, Lemma 2.1], this defines $\mathcal{C}$ as a graded Hopf algebra. Propositions 2.6 and 2.7 show that the linear map $\widetilde{F}: \mathcal{C} \rightarrow Q S y m$ induced by $\widetilde{F}$ is a morphism of graded Hopf algebras. (Compare [21, Proposition 4.4]; note that our $\widetilde{F}$ should not be confused with the similarly named function in [21].)

2.3. Properties of $\widetilde{\psi}_{u, v}$. Let $\mathbf{a}$ and $\mathbf{b}$ be two noncommuting indeterminates and $\mathbb{Z}\langle\mathbf{a}, \mathbf{b}\rangle$ be the ring of noncommutative polynomials in $\mathbf{a}$ and $\mathbf{b}$ with coefficients in $\mathbb{Z}$. Given $n \in \mathbb{P}$ and a subset $T \subseteq[n]$ let $m_{T}^{(n)}$ be the noncommutative monomial of degree $n$ in $\mathbf{a}$ and $\mathbf{b}$ whose $i$-th letter (from the left) is $\mathbf{a}$ if $i \notin T$ and $\mathbf{b}$ if $i \in T$, for $i=1, \ldots, n$. Given a Bruhat path $\Gamma=\left(u_{0}, u_{1}, \cdots, u_{k-1}, u_{k}\right)$ of length $k$ we define its weight to be $w(\Gamma) \stackrel{\text { def }}{=} m_{D(\Gamma)}^{(k-1)}$.

Proposition 2.9. Let $u, v \in W, u<v$. Then

$$
\widetilde{\psi}_{u, v}(\mathbf{a}+\mathbf{b}, \mathbf{a b}+\mathbf{b a})=\sum_{\Gamma} w(\Gamma)
$$

where $\Gamma$ runs over all the Bruhat paths from $u$ to $v$.

Proof. Let $w=\mathbf{c}^{n_{1}} \mathbf{d} \mathbf{c}^{n_{2}} \mathbf{d} \cdots \mathbf{c}^{n_{k}} \mathbf{d} \mathbf{c}^{n_{0}}$ be a cd-word $\left(n_{0}, \ldots, n_{k} \in \mathbb{N}, k \geq\right.$ $0)$. It then follows immediately from the definition of $B\left[\mathcal{I}^{w}\right]$ that

$$
(\mathbf{a}+\mathbf{b})^{n_{1}}(\mathbf{a b}+\mathbf{b a}) \cdots(\mathbf{a}+\mathbf{b})^{n_{k}}(\mathbf{a b}+\mathbf{b a})(\mathbf{a}+\mathbf{b})^{n_{0}}=\sum_{T \in B\left[\mathcal{I}^{w}\right]} m_{T}^{(|w|)}
$$

On the other hand, by Theorem 2.2 and Corollary 2.3,

$$
\widetilde{F}(u, v)=\sum_{\alpha} b_{\alpha}(u, v) L_{\alpha}=\sum_{w}[w]_{u, v} \Theta_{w}
$$

using (1.8) and equating coefficients of $L_{T}^{(n+1)}$ in (2.6) we obtain that

$$
b_{T}^{(n+1)}(u, v)=\sum_{\left\{w:|w|=n, T \in B\left[I^{w}\right]\right\}}[w]_{u, v},
$$

for all $n \in \mathbb{N}$ and $T \subseteq[n]$, where as in (1.3)

$$
b_{T}^{(n+1)}(u, v) \stackrel{\text { def }}{=}\left|\left\{\Gamma \in B_{n+1}(u, v): D(\Gamma)=T\right\}\right| .
$$


Therefore, by (2.5) and (2.7),

$$
\begin{aligned}
\widetilde{\psi}_{u, v}(\mathbf{a}+\mathbf{b}, \mathbf{a b}+\mathbf{b a}) & =\sum_{w}[w]_{u, v} \sum_{\left.T \in B_{[} \mathcal{I}^{w}\right]} m_{T}^{(|w|)} \\
& =\sum_{n \geq 0} \sum_{T \subseteq[n]}\left(\sum_{\left\{w:|w|=n, T \in B\left[I^{w}\right]\right\}}[w]_{u, v}\right) m_{T}^{(n)} \\
& =\sum_{n \geq 0} \sum_{T \subseteq[n]} b_{T}^{(n+1)}(u, v) m_{T}^{(n)} \\
& =\sum_{\Gamma} w(\Gamma),
\end{aligned}
$$

as desired.

From this we see that we can obtain the polynomial $\widetilde{R}_{u, v}$ directly from $\widetilde{\psi}_{u, v}$.

Corollary 2.10. For $u<v, \widetilde{R}_{u, v}(q)=q \widetilde{\psi}_{u, v}(q, 0)$.

Proof. From (2.7), one obtains $\left[\mathbf{c}^{n}\right]_{u, v}=b_{\emptyset}^{(n+1)}(u, v)$ for any $n$. Thus

$$
\begin{aligned}
q \widetilde{\psi}_{u, v}(q, 0) & =\sum_{n \geq 0}\left[\mathbf{c}^{n}\right]_{u, v} q^{n+1} \\
& =\sum_{\Gamma \in B(u, v): D(\Gamma)=\emptyset} q^{l(\Gamma)}=\widetilde{R}_{u, v}(q),
\end{aligned}
$$

the last equality being [7, Theorem 5.3.4].

Thus, referring to Example 2.4, we have $\widetilde{R}_{1234,4231}(q)=q^{5}+2 q^{3}+q$.

As in [23], we note that $\mathbb{Z}\langle\mathbf{a}, \mathbf{b}\rangle$ has a comultiplication defined for monomials by

$$
\Delta^{\prime}\left(a_{1} \cdots a_{n}\right) \stackrel{\text { def }}{=} \sum_{i=1}^{n} a_{1} \cdots a_{i-1} \otimes a_{i+1} \cdots a_{n}
$$

and extended linearly (where $\Delta^{\prime}(1) \stackrel{\text { def }}{=} 0 \otimes 0$ ). Similarly, we can define a second coproduct on Bruhat intervals by

$$
\Delta^{\prime}([u, v])=\sum_{u<z<v}[u, z] \otimes[z, v] .
$$

(Note that this is not the coproduct given in (2.2).) We define, for convenience,

$$
\widetilde{\phi}_{u, v}(\mathbf{a}, \mathbf{b}) \stackrel{\text { def }}{=} \widetilde{\psi}_{u, v}(\mathbf{a}+\mathbf{b}, \mathbf{a b}+\mathbf{b a}),
$$

for all $u, v \in W, u<v$. Then with the coalgebra structures just defined on Bruhat intervals and $\mathbb{Z}\langle\mathbf{a}, \mathbf{b}\rangle$, we have that $\widetilde{\phi}$ is a map of coalgebras. 
Proposition 2.11. Let $u, v \in W, u<v$. Then

$$
\Delta^{\prime}\left(\widetilde{\phi}_{u, v}\right)=\sum_{u<z<v} \widetilde{\phi}_{u, z} \otimes \widetilde{\phi}_{z, v}
$$

Proof. Given a Bruhat path $\Gamma=\left(u_{0}, u_{1}, \ldots, u_{k}\right)$ we let $\Gamma_{(i)} \stackrel{\text { def }}{=}\left(u_{0}, u_{1}, \ldots, u_{i}\right)$ and $\Gamma^{(i)} \stackrel{\text { def }}{=}\left(u_{i}, u_{i+1}, \ldots, u_{k}\right)$, for $i=1, \ldots, k-1$. By Proposition 2.9 and (2.8) we have that

$$
\begin{aligned}
\Delta^{\prime}\left(\widetilde{\phi}_{u, v}\right) & =\sum_{\Gamma} \Delta^{\prime}(w(\Gamma)) \\
& =\sum_{\Gamma} \sum_{i=1}^{l(\Gamma)-1} w\left(\Gamma_{(i)}\right) \otimes w\left(\Gamma^{(i)}\right) \\
& =\sum_{u<z<v} \sum_{\Gamma_{1} \in B(u, z)} \sum_{\Gamma_{2} \in B(z, v)} w\left(\Gamma_{1}\right) \otimes w\left(\Gamma_{2}\right) \\
& =\sum_{u<z<v} \widetilde{\phi}_{u, z} \otimes \widetilde{\phi}_{z, v},
\end{aligned}
$$

as desired.

If we let $\mathbf{c}=\mathbf{a}+\mathbf{b}$ and $\mathbf{d}=\mathbf{a b}+\mathbf{b a}$, then as in [23], the subalgebra $\mathbb{Z}\langle\mathbf{c}, \mathbf{d}\rangle$ of $\mathbb{Z}\langle\mathbf{a}, \mathbf{b}\rangle$ is closed under $\Delta^{\prime}$, since $\Delta^{\prime}(\mathbf{c})=2(1 \otimes 1)$ and $\Delta^{\prime}(\mathbf{d})=\mathbf{c} \otimes 1+1 \otimes \mathbf{c}$ $\left(\Delta^{\prime}\right.$ acts as a derivation on $\left.\mathbb{Z}\langle\mathbf{a}, \mathbf{b}\rangle\right)$. Thus $\widetilde{\psi}$ is a map of coalgebras:

$$
\Delta^{\prime}\left(\widetilde{\psi}_{u, v}\right)=\sum_{u<z<v} \widetilde{\psi}_{u, z} \otimes \widetilde{\psi}_{z, v}
$$

We note finally that the multiplicative structure used in [23], the poset join, does not carry over to Bruhat intervals. In most cases the join of two Bruhat intervals cannot be a Bruhat interval by [14, Theorem 3.2].

\section{KaZhdan-Lusztig POLYNomials AND the COMPlete cd-INDEX}

In this section we relate the Kazhdan-Lusztig polynomial of any Bruhat interval in an arbitrary Coxeter group to its complete cd-index.

Recall the definition of the polynomials $\Psi_{\alpha}$ and $\Upsilon_{\alpha}$ from $\S 1.1$. Consider the map $\mathcal{K}: Q S y m \rightarrow \mathbb{Z}\left[q^{1 / 2}, q^{-1 / 2}\right]$ defined by $\mathcal{K}\left(M_{\alpha}\right) \stackrel{\text { def }}{=}(-1)^{l(\alpha)} q^{-\frac{|\alpha|}{2}} \Psi_{\alpha}$ or, equivalently, $\mathcal{K}\left(L_{\alpha}\right) \stackrel{\text { def }}{=} q^{-\frac{|\alpha|}{2}} \Upsilon_{\alpha}$. Then by Theorem 2.2 we may rephrase Theorem 1.6 in the following way.

Proposition 3.1. Let $u, v \in W, u<v$. Then

$$
\mathcal{K}(\widetilde{F}(u, v))=q^{\frac{-l(u, v)}{2}} P_{u, v}(q)-q^{\frac{l(u, v)}{2}} P_{u, v}\left(\frac{1}{q}\right) .
$$

If we define $\Xi_{w} \stackrel{\text { def }}{=} \mathcal{K}\left(\Theta_{w}\right)$, then the following is immediate from Corollary 2.3 and Proposition 3.1 . 
Corollary 3.2. Let $u, v \in W, u<v$. Then

$$
q^{\frac{-l(u, v)}{2}} P_{u, v}(q)-q^{\frac{l(u, v)}{2}} P_{u, v}(1 / q)=\sum_{w}[w]_{u, v} \Xi_{w}
$$

We next give an explicit description of $\Xi_{w}$ in terms of $w$. Let $C_{i} \stackrel{\text { def }}{=}$ $\frac{1}{2 i+1}\left(\begin{array}{c}2 i+1 \\ i\end{array}\right)$ for $i \in \mathbb{N}$ be the $i$-th Catalan number, and set $C_{i}=0$ if $i \notin \mathbb{N}$. Let

$$
B_{k}(q) \stackrel{\text { def }}{=} \sum_{i=0}^{\lfloor k / 2\rfloor} \frac{k+1-2 i}{k+1}\left(\begin{array}{c}
k+1 \\
i
\end{array}\right) q^{i} .
$$

We call $B_{k}(q)$ the $k$-th ballot polynomial since it is closely related to ballot problems (see, e.g., [24, §III.1, p. 73]).

Let $a, b \in \mathbb{Z}, a \leq b$. By a lattice path on $[a, b]$ we mean a function $\Gamma:[a, b] \rightarrow \mathbb{Z}$ such that $\Gamma(a)=0$ and

$$
|\Gamma(i+1)-\Gamma(i)|=1
$$

for all $i \in[a, b-1]$. Given such a lattice path $\Gamma$ we let

$$
\begin{gathered}
N(\Gamma) \stackrel{\text { def }}{=}\{i \in[a+1, b-1]: \Gamma(i)<0\}, \\
d_{+}(\Gamma) \stackrel{\text { def }}{=}|\{i \in[a, b-1]: \Gamma(i+1)-\Gamma(i)=1\}|,
\end{gathered}
$$

$l(\Gamma) \stackrel{\text { def }}{=} b-a$, and $\Gamma_{\geq 0} \stackrel{\text { def }}{=} l(\Gamma)-1-|N(\Gamma)|$. We call $l(\Gamma)$ the length of $\Gamma$. Note that $b \notin N(\Gamma)$ and that

$$
d_{+}(\Gamma)=\frac{\Gamma(b)+b-a}{2} .
$$

We denote by $\mathcal{L}(n)$ the set of all lattice paths on $[0, n]$. From [12, Theorem 6.1] we have that for a composition $\alpha$,

$$
\Upsilon_{\alpha}(q)=(-1)^{|\alpha|-l(\alpha)} \sum_{\left\{\Gamma \in \mathcal{L}(|\alpha|): c o(N(\Gamma))=\alpha^{*}\right\}}(-q)^{d_{+}(\Gamma)} .
$$

For a set $T$, we denote by $T^{*}$ the set for which $\operatorname{co}\left(T^{*}\right)=\operatorname{co}(T)^{*}$.

The next result, along with the previous one, shows how one can compute the Kazhdan-Lusztig polynomial of any pair of elements $u, v \in W$ from the complete cd-index of the Bruhat interval $[u, v]$.

Theorem 3.3. Let $w=\mathbf{c}^{n_{0}} \mathbf{d} \mathbf{c}^{n_{1}} \mathbf{d} \cdots \mathbf{d} \mathbf{c}^{n_{k}}, k \geq 0, n_{0}, \ldots, n_{k} \geq 0$. Then

$$
\Xi_{w}=(-1)^{k+\frac{|w|-n_{0}}{2}}\left(q^{-\frac{n_{0}+1}{2}} B_{n_{0}}(-q)-q^{\frac{n_{0}+1}{2}} B_{n_{0}}\left(\frac{-1}{q}\right)\right) \prod_{j=1}^{k} C_{\frac{n_{j}}{2}} .
$$

In particular, $\Xi_{w}=0$ unless $n_{1} \equiv n_{2} \equiv \cdots \equiv n_{k} \equiv 0(\bmod 2)$. 
Proof. Let $n=|w|$, and for a cd-word $w=w_{1} \cdots w_{n}$, let $w^{*} \stackrel{\text { def }}{=} w_{n} \cdots w_{1}$. Note that $\left(\mathcal{I}^{w}\right)^{*}=\mathcal{I}^{w^{*}}$. Then, by (1.8) and (3.4) we have that

$$
\begin{aligned}
q^{\frac{n+1}{2}} \Xi_{w} & =\sum_{T \in B\left[\mathcal{I}^{w}\right]} \Upsilon_{c o(T)} \\
& =\sum_{T \in B\left[\mathcal{I}^{w}\right]} \sum_{\left\{\Gamma \in \mathcal{L}(n+1): N(\Gamma)^{*}=T\right\}}(-1)^{n+1-|N(\Gamma)|-1}(-q)^{d_{+}(\Gamma)} \\
& =\sum_{\left\{\Gamma \in \mathcal{L}(n+1), N(\Gamma), \overline{N(\Gamma)} \in b\left(\mathcal{I}^{w^{*}}\right)\right\}}(-1)^{\Gamma \geq 0}(-q)^{d_{+}(\Gamma)}
\end{aligned}
$$

Now, $\mathcal{I}^{w^{*}}=\left\{\left\{p_{1}-1, p_{1}\right\},\left\{p_{2}-1, p_{2}\right\}, \ldots,\left\{p_{k}-1, p_{k}\right\}\right\}$ where $p_{j} \stackrel{\text { def }}{=} n_{k+1-j}+$ $\cdots+n_{k}+2 j$ for $j \in[k]$, and clearly $\overline{N(\Gamma)}=\{i \in[n]: \Gamma(i) \geq 0\}$. Therefore $\Gamma \in \mathcal{L}(n+1)$ is such that $N(\Gamma) \cap\left\{p_{j}-1, p_{j}\right\} \neq \emptyset$ and $\overline{N(\Gamma)} \cap\left\{p_{j}-1, p_{j}\right\} \neq \emptyset$ for all $j \in[k]$ if and only if either $\Gamma\left(p_{j}-1\right)=\Gamma\left(p_{j}\right)-1=-1$ or $\Gamma\left(p_{j}-1\right)=$ $\Gamma\left(p_{j}\right)+1=0$, for all $j \in[k]$. Since $\Gamma$ is a lattice path this happens if and only if

$$
\Gamma\left(p_{j}-1\right)=\left\{\begin{array}{lll}
\Gamma\left(p_{j}\right)-1=-1, & \text { if } p_{j} \equiv 0 \quad(\bmod 2), \\
\Gamma\left(p_{j}\right)+1=0, & \text { if } p_{j} \equiv 1 \quad(\bmod 2),
\end{array}\right.
$$

for all $j \in[k]$.

Let, for brevity, $\mathcal{L}_{w}$ be the set of all lattice paths $\Gamma \in \mathcal{L}(n+1)$ that satisfy condition (3.6) so, by (3.5),

$$
q^{\frac{n+1}{2}} \Xi_{w}=\sum_{\Gamma \in \mathcal{L}_{w}}(-1)^{\Gamma \geq 0}(-q)^{d_{+}(\Gamma)} .
$$

We claim that

$$
q^{\frac{n+1}{2}} \Xi_{w}=\sum_{\Gamma \in \mathcal{L}_{w}^{*}}(-1)^{\Gamma \geq 0}(-q)^{d+(\Gamma)}
$$

where

$$
\mathcal{L}_{w}^{*} \stackrel{\text { def }}{=}\left\{\Gamma \in \mathcal{L}_{w}: \Gamma(i) \neq 0 \text { if } i \in[n+1] \backslash\left\{p_{1}, \ldots, p_{k}\right\}\right\} .
$$

In fact, let $\Gamma \in \mathcal{L}_{w} \backslash \mathcal{L}_{w}^{*}$ and let

$$
i_{0} \stackrel{\text { def }}{=} \min \left\{i \in[n+1] \backslash\left\{p_{1}, \ldots, p_{k}\right\}: \Gamma(i)=0\right\} .
$$

Note that $i_{0} \equiv 0(\bmod 2)$. Then $p_{r}<i_{0}<p_{r+1}$ for some $r \in[0, k]$ (where $p_{0} \stackrel{\text { def }}{=} 0$ and $\left.p_{k+1} \stackrel{\text { def }}{=} n+2\right)$. Since $\Gamma \in \mathcal{L}_{w}$ this implies that $\Gamma\left(p_{r}\right)=0$ (else, by (3.6), $\Gamma\left(p_{r}-1\right)=0$, contradicting the choice of $\left.i_{0}\right)$. Define $\Gamma^{\prime}:[0, n+1] \rightarrow \mathbb{Z}$ by

$$
\Gamma^{\prime}(i) \stackrel{\text { def }}{=} \begin{cases}-\Gamma(i), & \text { if } p_{r} \leq i \leq i_{0}, \\ \Gamma(i), & \text { otherwise }\end{cases}
$$

for $i \in[0, n+1]$. Then $\Gamma^{\prime} \in \mathcal{L}_{w} \backslash \mathcal{L}_{w}^{*}, d_{+}\left(\Gamma^{\prime}\right)=d_{+}(\Gamma)$ and $(-1)^{\Gamma_{\geq 0}^{\prime}}=$ $-(-1)^{\Gamma_{\geq 0}}$. It is clear that this map $\Gamma \mapsto \Gamma^{\prime}$ is an involution of $\mathcal{L}_{w} \backslash \mathcal{L}_{w}^{*}$ (since $i_{0}=\min \left\{i \in[n+1] \backslash\left\{p_{1}, \ldots, p_{k}\right\}: \Gamma^{\prime}(i)=0\right\}$ ) and that it has 
no fixed points, so $\sum_{\Gamma \in \mathcal{L}_{w} \backslash \mathcal{L}_{w}^{*}}(-1)^{\Gamma_{\geq 0}}(-q)^{d_{+}(\Gamma)}=0$ and (3.8) follows from (3.7).

Now let $\Gamma \in \mathcal{L}_{w}^{*}$. Then, by (3.9) and (3.6),

$$
\Gamma\left(p_{j}-1\right)=\Gamma\left(p_{j}\right)-1=-1
$$

and $p_{j} \equiv 0(\bmod 2)$ for all $j \in[k]$. This, again by (3.9)), implies that $\Gamma\left(p_{j-1}+\right.$ $1)=-1$ for all $j \in[k]$ and that $\Gamma(i) \leq-1$ for all $i \in\left[p_{k}\right] \backslash\left\{p_{1}, \ldots, p_{k}\right\}$. Therefore, by (3.8),

$$
\begin{aligned}
q^{\frac{n+1}{2}} \Xi_{w}= & \sum_{m=-n_{0}-1}^{n_{0}+1} \sum_{\left\{\Gamma \in \mathcal{L}_{w}^{*}: \Gamma(n+1)=m\right\}}(-1)^{\Gamma_{\geq 0}}(-q)^{d_{+}(\Gamma)} \\
= & \sum_{\left\{m \in\left[n_{0}+1\right]: m \equiv n_{0}+1\right.} \prod_{j=1}^{k} C_{\frac{n_{j}}{2}} \frac{m}{n_{0}+1}\left(\begin{array}{c}
n_{0}+1 \\
\frac{n_{0}+1-m}{2}
\end{array}\right) \\
& \left((-1)^{k}\left((-q)^{\frac{n+1+m}{2}}(-1)^{n_{0}}+(-q)^{\frac{n+1-m}{2}}\right)\right) \\
= & \sum_{i=0}^{\left\lfloor\frac{n_{0}}{2}\right\rfloor}(-1)^{k} \prod_{j=1}^{k} C_{\frac{n_{j}}{2}} \frac{n_{0}+1-2 i}{n_{0}+1}\left(\begin{array}{c}
n_{0}+1 \\
i
\end{array}\right) \\
& \left((-q)^{\frac{n+1+n_{0}+1-2 i}{2}}(-1)^{n_{0}}+(-q)^{\frac{n+1-n_{0}-1+2 i}{2}}\right) \\
= & (-1)^{k} \prod_{j=1}^{k} C_{\frac{n_{j}}{2}}(-q)^{\frac{n-n_{0}}{2}} \sum_{i=0}^{\left\lfloor\frac{n_{0}}{2}\right\rfloor} \frac{n_{0}+1-2 i}{n_{0}+1}\left(\begin{array}{c}
n_{0}+1 \\
i
\end{array}\right) \\
& \left((-q)^{n_{0}+1-i}(-1)^{n_{0}}+(-q)^{i}\right) \\
= & (-1)^{k} \prod_{j=1}^{k} C_{\frac{n_{j}}{2}}(-q)^{\frac{n-n_{0}}{2}}\left(B_{n_{0}}(-q)-q^{n_{0}+1} B_{n_{0}}(-1 / q)\right),
\end{aligned}
$$

where we have used (3.3) and well known results on lattice path enumeration (see, e.g., [34, Ex.6.20]).

\section{KAZHDAN-LUSZTig POLYNOMIALS AND BALLOT POLYNOMIALS}

Using the results in the previous section, we derive the expansion, in terms of the complete cd-index, of the Kazhdan-Lusztig polynomials with respect to a basis derived from the ballot polynomials (3.2). This basis and its relation to the Kazhdan-Lusztig polynomials was independently studied by Caselli [15, who also considered its relation to the $R$-polynomials.

In what follows, we will consider a fixed Bruhat interval $[u, v]$; the dependence on the pair $u, v$ will often be omitted. We set $n=l(u, v)-1$. For a cd-word $w=\mathbf{c}^{n_{0}} \mathbf{d} \mathbf{c}^{n_{1}} \mathbf{d} \cdots \mathbf{c}^{n_{k-1}} \mathbf{d} \mathbf{c}^{n_{k}}, k \geq 0, n_{0}, \ldots, n_{k} \geq 0$, define $C_{w} \stackrel{\text { def }}{=} \prod_{j=1}^{k} C_{\frac{n_{j}}{2}}$. If $w=\mathbf{c}^{m}$, we take $C_{w}=1$. Note that since $C_{i}=0$ when 
$i \notin \mathbb{N}, C_{w}=0$ unless $w$ is an even $\mathbf{c d}$-word, that is, unless $n_{1}, \ldots, n_{k}$ are all even.

It will be helpful to rework Corollary 3.2 to obtain an expression for the Kazhdan-Lusztig polynomial in terms of alternating shifted ballot polynomials $q^{i} B_{n-2 i}(-q)$. Note that $q^{i} B_{n-2 i}(-q)$ has degree $\lfloor n / 2\rfloor$ and lowest degree term $q^{i}$ with coefficient 1 . Thus the set of polynomials $q^{i} B_{n-2 i}(-q)$, $0 \leq i \leq\left\lfloor\frac{n}{2}\right\rfloor$, form a basis for the space of polynomials of degree $\leq\lfloor n / 2\rfloor$. Depending on the parity of $n$, Caselli denoted this basis by $O_{j}$ or $E_{j}$ (see [15, Theorem 6.5]).

We begin by deriving the expression for $P_{u, v}$ in terms of this basis as a function of the complete cd-index.

Theorem 4.1. Let $u, v \in W, u \leq v$. Then

$$
P_{u, v}(q)=\sum_{i=0}^{\lfloor n / 2\rfloor} a_{i} q^{i} B_{n-2 i}(-q),
$$

where

$$
a_{i}=a_{i}(u, v)=\left[\mathbf{c}^{n-2 i}\right]_{u, v}+\sum_{\mathbf{d} w \text { even }}(-1)^{\frac{|w|}{2}+|w|_{\mathbf{d}}} C_{\mathbf{d} w}\left[\mathbf{c}^{n-2 i} \mathbf{d} w\right]_{u, v} .
$$

Proof. Since $\operatorname{deg}\left(P_{u, v}\right) \leq\lfloor n / 2\rfloor$, it follows from Corollary 3.2 that

$$
P_{u, v}=\sum_{w}[w]_{u, v} D_{\frac{n}{2}}\left(q^{\frac{l(u, v)}{2}} \Xi_{w}\right)
$$

If $w=\mathbf{c}^{n_{0}} \mathbf{d} \mathbf{c}^{n_{1}} \mathbf{d} \mathbf{c}^{n_{2}} \cdots \mathbf{d} \mathbf{c}^{n_{k}}$, with $|w| \leq n=l(u, v)-1$, then by Theorem 3.3 .

$$
q^{\frac{l(u, v)}{2}} \Xi_{w}=(-1)^{k+\frac{|w|-n_{0}}{2}} C_{w}\left[q^{\frac{n-n_{0}}{2}} B_{n_{0}}(-q)-q^{\frac{n+n_{0}}{2}+1} B_{n_{0}}\left(\frac{-1}{q}\right)\right] .
$$

Thus, if $w$ is even and $[w]_{u, v} \neq 0$, then $n_{0} \equiv|w| \equiv n(\bmod 2)$ so

$$
D_{\frac{n}{2}}\left(q^{\frac{l(u, v)}{2}} \Xi_{w}\right)=(-1)^{k+\frac{|w|-n_{0}}{2}} C_{w} q^{\frac{n-n_{0}}{2}} B_{n_{0}}(-q),
$$

and (4.1) becomes

$$
P_{u, v}=\sum_{w \text { even }}(-1)^{|w|_{\mathbf{d}}+\frac{|w|-h(w)}{2}} C_{w}[w]_{u, v} q^{\frac{n-h(w)}{2}} B_{h(w)}(-q),
$$

where $h\left(\mathbf{c}^{n_{0}} \mathbf{d} \mathbf{c}^{n_{1}} \mathbf{d} \cdots \mathbf{c}^{n_{k-1}} \mathbf{d} \mathbf{c}^{n_{k}}\right) \stackrel{\text { def }}{=} n_{0}$ denotes the head of $w$.

Collecting the terms corresponding to cd words $w$ with $h(w)=n-2 i$ gives the statement of the theorem. 
Remark 4.2. Alternatively, we can write $a_{i}$ as a sum over subsets of $[i]$, namely

$$
\begin{gathered}
\sum_{\substack{S \subseteq[i] \\
S=\left\{i_{1}, \ldots, i_{k}\right\}<}}(-1)^{i_{k}-k}\left[\mathbf{c}^{n-2 i} \mathbf{d} \mathbf{c}^{2\left(i_{1}-1\right)} \mathbf{d c}^{2\left(i_{2}-i_{1}-1\right)} \ldots\right. \\
\left.\ldots \mathbf{d c}^{2\left(i_{k}-i_{k-1}-1\right)}\right]_{u, v} \prod_{j=1}^{k} C_{i_{j}-i_{j-1}-1} .
\end{gathered}
$$

Remark 4.3. We list the first few coefficients $a_{i}$ as functions of the complete cd-index (recall $n=l(u, v)-1$, and that we omit the dependence on $u, v$ ):

$$
\begin{aligned}
a_{0}= & {\left[\mathbf{c}^{n}\right] } \\
a_{1}= & {\left[\mathbf{c}^{n-2} \mathbf{d}\right]+\left[\mathbf{c}^{n-2}\right] } \\
a_{2}= & {\left[\mathbf{c}^{n-4} \mathbf{d}^{2}\right]-\left[\mathbf{c}^{n-4} \mathbf{d} \mathbf{c}^{2}\right]+\left[\mathbf{c}^{n-4} \mathbf{d}\right]+\left[\mathbf{c}^{n-4}\right] } \\
a_{3}=\left[\mathbf{c}^{n-6} \mathbf{d}^{3}\right]- & {\left[\mathbf{c}^{n-6} \mathbf{d}^{2} \mathbf{c}^{2}\right]-\left[\mathbf{c}^{n-6} \mathbf{d} \mathbf{c}^{2} \mathbf{d}\right]+2\left[\mathbf{c}^{n-6} \mathbf{d} \mathbf{c}^{4}\right] } \\
& +\left[\mathbf{c}^{n-6} \mathbf{d}^{2}\right]-\left[\mathbf{c}^{n-6} \mathbf{d} \mathbf{c}^{2}\right]+\left[\mathbf{c}^{n-6} \mathbf{d}\right]+\left[\mathbf{c}^{n-6}\right] .
\end{aligned}
$$

Remark 4.4. By Proposition 2.5, the degree $n$ terms of the complete cdindex of the Bruhat interval $[u, v]$ correspond to the ordinary cd-index of the Eulerian poset $[u, v]$. Restricting the formulas for the $a_{i}$ in Theorem 4.1 to only the degree $n$ cd-coefficients yields the expression given by Bayer and Ehrenborg [3, Theorem 4.2] for the $g$-polynomial $g\left([u, v]^{*}, q\right)$ in terms of the cd-index of the dual interval $[u, v]^{*}$. This can be checked by comparing formulas - for example the polynomial $Q_{k+1}(x)$ of [3] is $B_{k}(-x)-$ and recalling that $[w]_{u, v}=\left[w^{*}\right]_{[u, v]^{*}}$. One consequence is that the difference $P_{u, v}(q)-g\left([u, v]^{*}, q\right)$ is a function of the lower degree cd-coefficients only.

Example 4.5. Continuing with Example 2.4, we have

$$
\widetilde{F}(1234,4231)=\Theta_{\mathbf{c}^{4}}+\Theta_{\mathbf{d c}^{2}}+2 \Theta_{\mathbf{c d c}}+2 \Theta_{\mathbf{c}^{2} \mathbf{d}}+2 \Theta_{\mathbf{d}^{2}}+2 \Theta_{\mathbf{c}^{2}}+\Theta_{\mathbf{1}}
$$

and so

$$
\begin{aligned}
P_{u, v}(q)= & a_{0} q^{0} B_{4}(-q)+a_{1} q^{1} B_{2}(-q)+a_{2} q^{2} B_{0}(-q) \\
= & {\left[\mathbf{c}^{4}\right]\left(1-3 q+2 q^{2}\right)+\left(\left[\mathbf{c}^{2} \mathbf{d}\right]+\left[\mathbf{c}^{2}\right]\right) q(1-q) } \\
& \quad+\left(\left[\mathbf{d}^{2}\right]-\left[\mathbf{d} \mathbf{c}^{2}\right]+[\mathbf{d}]+[\mathbf{1}]\right) q^{2} \\
= & \left(1-q+q^{2}\right)+\left(2 q-q^{2}\right)=1+q
\end{aligned}
$$

with $g\left([u, v]^{*}, q\right)=1-q+q^{2}$.

Next we give an example that shows that it is not possible to express $P_{u, v}$ as a function of the ordinary (homogeneous) cd-index $\psi_{u, v}$ alone. That is, the Kazhdan-Lusztig polynomial does not depend only on the flag $f$-vector of the Eulerian poset $[u, v]$. 
Example 4.6. Let $W=S_{5}$ and consider the rank 6 Bruhat intervals $[12435,53142]$ and $[31254,53421]$. One can compute

$$
\begin{aligned}
\widetilde{\psi}_{12435,53142}=\mathbf{c}^{5} & +6 \mathbf{c} \mathbf{d} \mathbf{c}^{2}+6 \mathbf{c}^{2} \mathbf{d} \mathbf{c}+3 \mathbf{d} \mathbf{c}^{3}+3 \mathbf{c}^{3} \mathbf{d}+7 \mathbf{c} \mathbf{d}^{2}+7 \mathbf{d}^{2} \mathbf{c}+6 \mathbf{d} \mathbf{c d} \\
& +\mathbf{c}^{3}+2 \mathbf{d} \mathbf{c}+2 \mathbf{c d} \\
\widetilde{\psi}_{31254,53421}=\mathbf{c}^{5} & +6 \mathbf{c d} \mathbf{c}^{2}+6 \mathbf{c}^{2} \mathbf{d} \mathbf{c}+3 \mathbf{d} \mathbf{c}^{3}+3 \mathbf{c}^{3} \mathbf{d}+7 \mathbf{c} \mathbf{d}^{2}+7 \mathbf{d}^{2} \mathbf{c}+6 \mathbf{d} \mathbf{c d} \\
& +2 \mathbf{c}^{3}+4 \mathbf{d} \mathbf{c}+4 \mathbf{c d}
\end{aligned}
$$

while $P_{12435,53142}=1$ and $P_{31254,53421}=1+q$. Thus neither $\widetilde{\psi}_{u, v}$ nor $P_{u, v}$ is a function of $\psi_{u, v}$ alone.

It appears that no such example was previously known. It can be checked that there is no such pair of intervals in $S_{4}$. There are many other examples in $S_{5}$, although none of them involve lower intervals $(i . e$., those with $u=e$ ).

Considering the dependence of the coefficients of the Kazhdan-Lusztig polynomials on these coefficients $a_{i}$, let $P_{u, v}=p_{0}+p_{1} q+\cdots$. The following is a direct consequence of the definition of the $a_{i}$ as the coefficients of $P_{u, v}$ in the basis $q^{i} B_{n-2 i}(-q)$. Propositions 4.7 and 4.8 as well as Corollary 4.9 are all implicit in [15, §6], so proofs will be omitted here.

Proposition 4.7. For $j=0, \ldots,\lfloor n / 2\rfloor$,

$$
p_{j}=\sum_{i=0}^{j}(-1)^{j-i} \frac{n+1-2 j}{n+1-2 i}\left(\begin{array}{c}
n+1-2 i \\
j-i
\end{array}\right) a_{i} .
$$

Theorem 4.1 and Proposition 4.7 allow us to derive the coefficients of the Kazhdan-Lusztig polynomial for any Bruhat interval as a function of its complete cd-index. For example, we can read from Proposition 4.7 and (4.3) that

$$
\begin{aligned}
p_{1}=[q]\left(P_{u, v}\right) & =a_{1}-(n-1) a_{0} \\
& =\left[\mathbf{c}^{n-2} \mathbf{d}\right]+\left[\mathbf{c}^{n-2}\right]-(n-1)\left[\mathbf{c}^{n}\right] .
\end{aligned}
$$

The relations given in Proposition 4.7 are unitriangular so invertible. The inverse relations have a particularly simple nonnegative form.

Proposition 4.8. For $j=0, \ldots,\lfloor n / 2\rfloor$,

$$
a_{j}=\sum_{i=0}^{j}\left(\begin{array}{c}
n-j-i \\
n-2 j
\end{array}\right) p_{i} .
$$

Corollary 4.9. Nonnegativity of $a_{i}(u, v), i=1, \ldots, k$ is implied by the nonnegativity of $\left[q^{i}\right]\left(P_{u, v}\right), i=1, \ldots, k$.

Remark 4.10. In fact, any nonnegative polynomial of degree $\leq\left\lfloor\frac{n}{2}\right\rfloor$ has a nonnegative representation in terms of the basis of alternating shifted ballot polynomials $q^{i} B_{n-2 i}(-q)$. 
Given the conjectured nonnegativity of the coefficients of the KazhdanLusztig polynomial $P_{u, v}$ for any Bruhat interval $[u, v]$ [27], Caselli made the following conjecture. Because of Theorem 4.1 (see also (4.3)), we can interpret it as a conjectured set of linear inequalities that must be satisfied by the complete cd-index.

Conjecture 4.11. [15, Conjecture 6.6] For each Bruhat interval $[u, v]$ and for each $i=0,1, \ldots,\lfloor n / 2\rfloor, n=l(u, v)-1$, we have $a_{i}(u, v) \geq 0$.

We conclude by noting the following consequence of Proposition 4.8 ,

Corollary 4.12. Let $u, v \in W, u<v$. Then

$$
a_{\left\lfloor\frac{l(u, v)-1}{2}\right\rfloor}= \begin{cases}P_{u, v}(1), & l(u, v) \text { odd } \\ \left.\frac{d}{d q}\left(q^{\frac{l(u, v)}{2}} P_{u, v}(1 / q)\right)\right|_{q=1}, & l(u, v) \text { even. }\end{cases}
$$

Remark 4.13. An interesting question is whether, for all $w$, the quantities $[w]_{u, v}$ are combinatorially invariant, that is, they depend only on the poset structure of the interval $[u, v]$. This is true for cd-words $w$ of degree $l(u, v)-1$ by Proposition 2.5. By Theorem 4.1, combinatorial invariance of $[w]_{u, v}$ for all $w$ implies the conjectured combinatorial invariance of the Kazhdan-Lusztig polynomial $P_{u, v}$. But the converse is also true: if $P_{u, v}$ is combinatorially invariant for all $u<v$, then so is $[w]_{u, v}$ for all $w$ and all $u<v$. This follows from Proposition 1.5 and Theorem 1.4 and the fact that $[w]_{u, v}$ depends on the $c_{\alpha}$, by (1.4) and Proposition 5.1. Combinatorial invariance of $P_{u, v}$ is known to hold in the case $u=e$ (see [29], 14] and [17]), and it follows that the same is true for all $[w]_{e, v}$.

\section{Other REPRESENTATIONS OF THE COMPLETE cd-INDEX}

The formulas obtained in the previous sections for the Kazhdan-Lusztig polynomials assume knowledge of the complete cd-index. In this section we show how one can explicitly compute the complete cd-index of a Bruhat interval $[u, v]$ in terms of the coefficients $b_{\alpha}(u, v)$ defined in $\S 1.1$.

Let $u, v \in W, u<v$, and $n \in[l(u, v)-1], n \equiv l(u, v)-1(\bmod 2)$. In this section it will be convenient to index the $b_{\alpha}$ by subsets instead of the corresponding compositions. Unless otherwise explicitly stated, all subsets are in $[n]$. We will omit to write the dependence on $u, v$ throughout the section.

We start with a general expression for $[w]$ in terms of sparse $b_{S}$, that is, those $b_{S}, S \subseteq[n]$, where $S$ has no two successive elements and $n \notin S$. In terms of $b_{\alpha}$, this means that $\alpha_{i}>1$ if $i>1$. We use an expression for $[w]$ in terms of the sparse $k$-vector, which is a reinversion of the sparse $b_{S}$ defined by $k_{S}=\sum_{T \subseteq S}(-1)^{|S|-|T|} b_{T}$. The following result is proved in exactly the same way as [5, Proposition 7.1]. 
Proposition 5.1. Let $w=\mathbf{c}^{n_{1}} \mathbf{d} \mathbf{c}^{n_{2}} \mathbf{d} \cdots \mathbf{c}^{n_{k}} \mathbf{d c}^{n_{0}}$ and define $m_{0}, \ldots, m_{k}$ by $m_{0}=1$ and $m_{i}=m_{i-1}+n_{i}+2$. Then

$$
[w]=\sum_{i_{1}, \ldots, i_{k}}(-1)^{\left(m_{1}-i_{1}\right)+\left(m_{2}-i_{2}\right)+\cdots+\left(m_{k}-i_{k}\right)} k_{\left\{i_{1} i_{2} \cdots i_{k}\right\}},
$$

where the sum is over all $k$-tuples $\left(i_{1}, i_{2}, \ldots, i_{k}\right)$ such that $m_{j-1} \leq i_{j} \leq$ $m_{j}-2$.

The inversion of this relation, expressing $k_{S}$ as a sum of distinct $[w]$, is the same as [6, Proposition 2.3]. Using Proposition 5.1 one can obtain from (4.4) the coefficient of the linear term of the Kazhdan-Lusztig polynomial $P_{u, v}$ (compare [10, Corollary 5.9] and [8, Theorem D]).

Corollary 5.2. For any Bruhat interval $[u, v]$, the coefficient of the linear term in $P_{u, v}$ is

$$
p_{1}=c_{n, 1}(u, v)+c_{n-1}(u, v)-(n+1) .
$$

We will show that if $[w]$ is expressed in terms of the sparse $b_{S}$, the coefficients are still \pm 1 , leading to the possibility of a direct enumerative interpretation of $[w]$. Using the notation of Proposition 5.1, let

$$
A_{j}=\left\{i: m_{j-1} \leq i \leq m_{j}-2\right\}
$$

be the range of $i_{j}$ above, and let

$$
\mathcal{T}_{w} \stackrel{\text { def }}{=}\left\{T \subset \cup_{i \leq k} A_{i}:\left|T \cap A_{j}\right| \leq 1 \forall j\right\} .
$$

Then $k_{S}$ appears in (5.1) if and only if $S \in \mathcal{T}_{w}$ and $|S|=k$, and by Proposition 5.1 and the definition of $k_{S}$, we see that $[w]=\sum_{S \in \mathcal{T}_{w}} d_{S} b_{S}$ for integers $d_{S}$. We now prove that these integers can only be \pm 1 or 0 .

Proposition 5.3. If $w=\mathbf{c}^{n_{1}} \mathbf{d} \cdots \mathbf{c}^{n_{k}} \mathbf{d} \mathbf{c}^{n_{0}}$, then $[w]=\sum_{S \in \mathcal{T}_{w}} d_{S} b_{S}$, where

$$
d_{S}= \begin{cases}(-1)^{k-|S|} \prod_{j} \prod_{i \in S \cap A_{j}}(-1)^{m_{j}-i}, & \text { if }\left|S \cap A_{i}\right|=1 \text { for }\left|A_{i}\right| \text { even, } \\ 0, & \text { otherwise. }\end{cases}
$$

Proof. By (5.1) and the definition of $k_{S}$ we have

$$
\begin{aligned}
d_{S} & =\sum_{S \subseteq\left\{i_{1}, \ldots, i_{k}\right\} \in \mathcal{T}_{w}}(-1)^{k-|S|}(-1)^{\left(m_{1}-i_{1}\right)+\left(m_{2}-i_{2}\right)+\cdots+\left(m_{k}-i_{k}\right)} \\
& =(-1)^{k-|S|}\left[\prod_{j: S \cap A_{j} \neq \emptyset} \prod_{i \in S \cap A_{j}}(-1)^{m_{j}-i}\right]\left[\prod_{j: S \cap A_{j}=\emptyset}\left(\sum_{i \in A_{j}}(-1)^{m_{j}-i}\right)\right] .
\end{aligned}
$$

But the sums in the last expression are 0 or 1 depending on whether $\left|A_{j}\right|$ are even or odd, so the result follows.

For example, for $w=\mathbf{c d}^{2}, k=2, n_{1}=1, n_{2}=0, n_{0}=0, A_{1}=\{1,2\}$, $A_{2}=\{4\}$ and so

$$
\left[\mathbf{c d}^{2}\right]=b_{\{2,4\}}-b_{\{1,4\}}-b_{\{2\}}+b_{\{1\}} .
$$


Note that the same expression computes the coefficient $[w]=\left[\mathbf{c d}^{2} \mathbf{c}^{m}\right]$ for any $m \geq 0$, where the subscripts are understood to be subsets of $\{1, \ldots, \operatorname{deg} w\}$.

Finally, we give simple proofs of two elementary identities between cdcoefficients and the numbers $b_{\alpha}$ using basic quasisymmetric identities. The second of these is essentially in [33].

Proposition 5.4. Let $u, v \in W, u<v$, and $n \in[l(u, v)-1], n \equiv l(u, v)-1$ $(\bmod 2)$. Then:

(1) $\sum_{w} 2^{n-|w|_{d}}[w]_{u, v}=c_{[n]}(u, v)$, the number of Bruhat paths from $u$ to $v$ of length $n+1$,

(2) $\sum_{w}[w]_{u, v}=b_{\{1,3,5, \ldots\}}(u, v)$,

where the sums are over all cd-words of degree $n$.

Proof. By Theorem 2.2

$$
\widetilde{F}_{n+1}(u, v)=\sum_{S} c_{S}(u, v) M_{S}^{(n+1)}=\sum_{S} b_{S}(u, v) L_{S}^{(n+1)}=\sum_{w}[w]_{u, v} \Theta_{w},
$$

where the sums are over all subsets of $[n]$ and over all cd-words of degree $n$ (recall that we denote by $\widetilde{F}_{i}$ the homogeneous component of $\widetilde{F}$ of degree $i$ ). We now use the relations

$$
\Theta_{w}=\sum_{S \in b\left[\mathcal{I}_{w}\right]} 2^{|S|-|w| \mathbf{d}} M_{S}^{(n+1)}=\sum_{T \in B\left[\mathcal{I}^{w}\right]} L_{T}^{(n+1)}
$$

from [6, (2.14)] (divided, as before, by $2^{|w|_{\mathbf{d}}+1}$ ) and (1.8). Hence the coefficient of $M_{[n]}^{(n+1)}$ in $\Theta_{w}$ is $2^{n-|w|_{\mathbf{d}}}$, so we can conclude (1). Similarly, the coefficient of $L_{\{1,3,5, \ldots\}}$ in $\Theta_{w}$ is 1 , so (2) follows.

\section{A Sign COnjecture For the COMPlete cd-IndeX}

We conclude with an intriguing sign conjecture for the complete cd-index and prove one of its simple consequences in certain cases. Computation on Bruhat intervals up to rank 7 occurring in symmetric groups suggests the following.

Conjecture 6.1. Let $(W, S)$ be a Coxeter system and $u, v \in W, u<v$. Then

$$
[w]_{u, v} \geq 0
$$

for all cd-words $w$.

Since every Bruhat interval is shellable (e.g., [7, Theorem 2.7.5]) and Eulerian, hence Gorenstein*, it follows from the recent work of Karu [26] (see also [22]) that $[w]_{u, v} \geq 0$ whenever $\operatorname{deg} w=l(u, v)-1$ (the top degree for which $\left.[w]_{u, v} \neq 0\right)$.

If Conjecture 6.1 were true, then Proposition 5.4 would imply that

$$
2^{n}\left[\mathbf{c}^{n}\right]_{u, v} \leq c_{[n]}(u, v)
$$


for all $u, v \in W$ and $n \geq 0$. This is indeed true for finite Coxeter and affine Weyl groups, as we now show. From now on, we assume that $\mathrm{W}$ is a finite Coxeter or affine Weyl group.

Given two Bruhat paths $\Delta=(u, x, v), \Gamma=(u, y, v) \in B_{2}(u, v)$ write $\Delta \leq \Gamma$ if $\left(x u^{-1}, v x^{-1}\right)$ is lexicographically smaller than $\left(y u^{-1}, v y^{-1}\right)$ (where $T$ is totally ordered by the chosen reflection ordering). Let $m \stackrel{\text { def }}{=}\left[t^{2}\right]\left(\widetilde{R}_{u, v}\right)$. It then follows easily from [7, Theorem 5.3.4] that $\left|B_{2}(u, v)\right|=2 \mathrm{~m}$ and that

$$
\left|\left\{\Delta \in B_{2}(u, v): D(\Delta)=\emptyset\right\}\right|=m=\left|\left\{\Delta \in B_{2}(u, v): D(\Delta)=\{1\}\right\}\right| .
$$

For $\Gamma \in B_{2}(u, v)$ let

$$
r \stackrel{\text { def }}{=}\left|\left\{\Delta \in B_{2}(u, v): D(\Delta)=D(\Gamma), \Delta \leq \Gamma\right\}\right| .
$$

The flip of $\Gamma$ is the $r$-th Bruhat path (in the lexicographic ordering) in $\left\{\Delta \in B_{2}(u, v): D(\Delta) \neq D(\Gamma)\right\}$. We denote this path by flip $(\Gamma)$. This notion is a special case of that of lexicographic correspondence first introduced in [13. The following result is a special case of [13, Corollary 2.3].

Proposition 6.2. Let $W$ be a finite Coxeter or affine Weyl group. Further, let $u, v \in W, u<v,(u, y, v) \in B_{2}(u, v)$ be such that $D((u, y, v))=\emptyset$ and $(u, x, v) \stackrel{\text { def }}{=}$ flip $((u, y, v))$. Then $y u^{-1}<_{T} x u^{-1}$ and $v x^{-1}<_{T} v y^{-1}$.

Note that $f \operatorname{lip}(f \operatorname{fip}(\Gamma))=\Gamma$. Given $\Delta=\left(u_{0}, u_{1}, \ldots, u_{k}\right) \in B_{k}(u, v)$ we let, for $i \in[k-1]$, flip $(\Delta)$ be the Bruhat path in $B_{k}(u, v)$ obtained by flipping $\Delta$ at its $i$-th element. Namely, flip $i(\Delta) \stackrel{\text { def }}{=}\left(u_{0}, u_{1}, \ldots, u_{i-1}\right.$, $\left.x, u_{i+1}, \ldots, u_{k-1}, u_{k}\right)$ where $\left(u_{i-1}, x, u_{i+1}\right) \stackrel{\text { def }}{=} \operatorname{flip}\left(\left(u_{i-1}, u_{i}, u_{i+1}\right)\right)$. Note that if $\{i-1, i, i+1\} \cap D(\Delta)=\emptyset$ then, by Proposition 6.2. $\{i-1, i, i+1\} \cap$ $D\left(\operatorname{flip}_{i}(\Delta)\right)=\{i\}$.

Proposition 6.3. Let $W$ be a finite Coxeter or affine Weyl group and $u, v \in$ $W, u<v$. Then

$$
\left[t^{k}\right]\left(\widetilde{R}_{u, v}\right) \leq\left\lfloor\frac{\left|B_{k}(u, v)\right|}{2^{k-1}}\right\rfloor
$$

for all $k \geq 1$, and so $2^{n}\left[\mathbf{c}^{n}\right]_{u, v} \leq c_{[n]}(u, v)$ for $n \geq 0$.

Proof. Since $\left[t^{k}\right]\left(\widetilde{R}_{u, v}\right) \in \mathbb{N}$, we prove the equivalent statement that

$$
\left[t^{k}\right]\left(\widetilde{R}_{u, v}\right) 2^{k-1} \leq\left|B_{k}(u, v)\right| .
$$

We do this by constructing an explicit injection.

Let $\Gamma=\left(u, u_{1}, \cdots, u_{k-1}, v\right) \in B_{k}(u, v)$ be such that $D(\Gamma)=\emptyset$ and $S=$ $\left\{s_{1}, \ldots, s_{r}\right\}_{<} \subseteq[k-1]$. We define

$$
\varphi(\Gamma, S) \stackrel{\text { def }}{=} \operatorname{flip}_{s_{r}}\left(\cdots\left(\text { flip }_{s_{1}}(\Gamma)\right) \cdots\right) .
$$

Clearly, $\varphi(\Gamma, S) \in B_{k}(u, v)$. Furthermore, note that by Proposition 6.2

$$
\begin{aligned}
s_{i} & =\max \left\{D\left(\operatorname{flip}_{s_{i}}\left(\cdots\left(\operatorname{flip}_{s_{1}}(\Gamma)\right) \cdots\right)\right)\right\} \\
& =\max \left\{D\left(\operatorname{flip}_{s_{i+1}}\left(\cdots\left(\operatorname{flip}_{s_{r}}(\varphi(\Gamma, S))\right) \cdots\right)\right)\right\}
\end{aligned}
$$


for $i=1, \ldots, r$. Therefore $s_{r}, \ldots, s_{1}$ and hence $\Gamma$ are uniquely recoverable from $\varphi(\Gamma, S)$, so $\varphi$ is an injection. The result now follows from the fact that $\left[t^{n+1}\right]\left(\widetilde{R}_{u, v}\right)=\left[\mathbf{c}^{n}\right]_{u, v}$ by Corollary 2.10 .

Remark 6.4. We note that Proposition 6.3 holds whenever Proposition 6.2 does, and the latter has been conjectured to hold for all Coxeter groups (see [16, p. 117] and [13, p. 745]), giving further evidence for Conjecture 6.1, In fact, a proof of Proposition 6.2 that holds for all Coxeter groups has been recently announced $[9]$.

\section{REFERENCES}

[1] M. Aguiar, N. Bergeron, and F. Sottile, Combinatorial Hopf algebras and generalized Dehn-Sommerville relations, Compos. Math. 142 (2006), 1-30.

[2] M.M. Bayer and L.J. Billera, Generalized Dehn-Sommerville relations for polytopes, spheres and Eulerian partially ordered sets, Inventiones Math. 79 (1985) 143-157.

[3] M.M. Bayer and R. Ehrenborg, The toric $h$-vectors of partially ordered sets, Trans. Amer. Math. Soc. 352 (2000), 4515-4531.

[4] M.M. Bayer and A. Klapper, A new index for polytopes, Discrete Comput. Geometry 6 (1991), 33-47.

[5] L.J. Billera and R. Ehrenborg, Monotonicity of the cd-index for polytopes, Math. Z. 233 (2000), 421-441.

[6] L.J. Billera, S. K. Hsiao, and S. van Willigenburg, Peak quasisymmetric functions and Eulerian enumeration, Adv. Math. 176 (2003), no. 2, 248-276.

[7] A. Björner and F. Brenti, Combinatorics of Coxeter Groups, Graduate Texts in Mathematics 231, Springer, New York, 2005.

[8] A. Björner and T. Ekedahl, On the shape of Bruhat intervals, Annals of Math. 170 (2009), 799-817.

[9] S. A. Blanco, private communication, 2008.

[10] F. Brenti, A combinatorial formula for Kazhdan-Lusztig polynomials, Inventiones Math. 118 (1994), 371-394.

[11] F. Brenti, Combinatorial expansions of Kazhdan-Lusztig polynomials, J. London Math. Soc. 55 (1997), 448-472.

[12] F. Brenti, Lattice paths and Kazhdan-Lusztig polynomials, Jour. Amer. Math. Soc. 11 (1998), 229-259.

[13] F. Brenti and F. Incitti, Lattice paths, lexicographic correspondence and KazhdanLusztig polynomials, J. Algebra 303 (2006), 742-762.

[14] F. Brenti, F. Caselli, and M. Marietti, Special matchings and Kazhdan-Lusztig polynomials, Advances in Math. 202 (2006), 555-601.

[15] F. Caselli, Non-neegativity properties of $R$-polynomials, European J. Combin. 27 (2006), 1005-1021.

[16] P. Cellini, T-increasing paths on the Bruhat graph of affine Weyl groups are selfavoiding, J. Algebra, 228 (2000), 107-118.

[17] E. Delanoy, Combinatorial invariance of Kazhdan-Lusztig polynomials on intervals starting from the identity, J Algebr Comb 24 (2006), 437-463.

[18] V. Deodhar, On some geometric aspects of Bruhat orderings. I. A finer decomposition of Bruhat cells. Inventiones Math. 79 (1985), 499-511.

[19] M. Dyer, On the "Bruhat graph" of a Coxeter system, Compos. Math., 78 (1991), 185-191.

[20] M. Dyer, Hecke algebras and shellings of Bruhat intervals, Compos. Math., 89 (1993), 91-115.

[21] R. Ehrenborg, On posets and Hopf algebras, Adv. in Math. 119 (1996), 1-25. 
[22] R. Ehrenborg and K. Karu, Decomposition theorem for the cd-index of Gorenstein* posets, J. Algebr. Comb. 26 (2007), 225-251.

[23] R. Ehrenborg and M. Readdy, Coproducts and the cd-index, J. Algebraic Combinatorics 8 (1998), 273-299.

[24] W. Feller, An Introduction to Probability Theory and its Applications, Vol. 1, Wiley, New York, 1950.

[25] J. E. Humphreys, Reflection Groups and Coxeter Groups, Cambridge University Press, Cambridge, 1990.

[26] K. Karu, The cd-index of fans and posets, Compos. Math., 142 (2006), 701-718.

[27] D. Kazhdan and G. Lusztig, Representations of Coxeter groups and Hecke algebras, Inventiones Math. 53 (1979), 165-184.

[28] D. Kazhdan, G. Lusztig, Schubert varieties and Poincaré duality, Geometry of the Laplace operator, Proc. Sympos. Pure Math. 34, Amer. Math. Soc., Providence, RI, 1980, pp. 185-203.

[29] M. Marietti, Kazhdan-Lusztig theory: Boolean elements, special matchings and combinatorial invariance, Ph.D. thesis, Universita' di Roma "La Sapienza", Roma, 2003.

[30] N. Reading, The cd-index of Bruhat intervals., Electron. J. Combin. 11 (2004), no. 1, Research Paper 74, 25 pp. (electronic).

[31] R. P. Stanley, Generalized h-vectors, intersection cohomology of toric varieties, and related results, Adv. Studies Pure Math., 11 (1987), 187-213.

[32] R.P. Stanley, Subdivisions and local h-vectors, Jour. Amer. Math. Soc. 5 (1992), 805-851.

[33] R.P. Stanley, Flag $f$-vectors and the $c d$-index, Math. Z. 216 (1994), 483-499.

[34] R. Stanley, Enumerative Combinatorics, Vol. 2, Cambridge Studies in Advanced Mathematics, Vol. 62, Cambridge University Press, Cambridge, UK, 1999.

[35] J.R. Stembridge, Enriched P-partitions, Trans. Amer. Math. Soc. 349 no. 2 (1997), 763-788.

Department of Mathematics, Cornell University, Ithaca, NY 14853-4201

E-mail address: billera@math.cornell.edu

Dipartimento di Matematica, Universitá di Roma “Tor Vergata”, Via della

Ricerca Scientifica, 1, I-00133 Roma, Italy

E-mail address: brenti@mat.uniroma2.it 